\title{
Comparison of Different Confidence Intervals of Intensities for an Open Queueing Network with Feedback
}

\author{
Vinayak Kawaduji Gedam ${ }^{1}$, Suresh Bajirao Pathare ${ }^{2}$ \\ ${ }^{1}$ Department of Statistics, University of Pune, Pune, India \\ ${ }^{2}$ Indira College of Commerce and Science, Pune, India \\ Email: vkgedam@stats.unipune.ac.in, sureshpathare_1975@yahoo.com
}

Received March 14, 2012; revised April 30, 2012; accepted May 10, 2012

\begin{abstract}
In this paper we propose a consistent and asymptotically normal estimator (CAN) of intensities $\rho_{1}, \rho_{2}$ for a queueing network with feedback (in which a job may return to previously visited nodes) with distribution-free inter-arrival and service times. Using this estimator and its estimated variance, some $100(1-\alpha) \%$ asymptotic confidence intervals of intensities are constructed. Also bootstrap approaches such as Standard bootstrap, Bayesian bootstrap, Percentile bootstrap and Bias-corrected and accelerated bootstrap are also applied to develop the confidence intervals of intensities. A comparative analysis is conducted to demonstrate performances of the confidence intervals of intensities for a queueing network with short run data.
\end{abstract}

Keywords: Coverage Percentage; Relative Coverage; Bayesian Bootstrap; Bias-Corrected and Accelerated Bootstrap; Percentile Bootstrap; Standard Bootstrap

\section{Introduction}

Consider a queueing network of a computer system with feedback (in which a job may return to previously visited nodes) as shown in Figure 1. This queueing network consists of a CPU node and an Input/Output (I/O) node. External jobs arrive at the CPU node according to the rate $\lambda$. After service completion at CPU node, the job proceeds to the I/O node with probability $p_{1}$, and with probability $p_{0}$ the job departs from the system, where $p_{0}=1-p_{1}$. Jobs leaving the $\mathrm{I} / \mathrm{O}$ node are always fed back to the CPU node (see Figure 1). The service times at each node are with rates $\mu_{1}$ and $\mu_{2}$ respectively. The successive service times at both nodes are assumed to be mutually independent and independent of the state of the system. The traffic intensity at the CPU node and I/O node is given by

$$
\rho_{1}=\frac{\lambda}{p_{0} \mu_{1}}, \rho_{2}=\frac{p_{1} \lambda}{p_{0} \mu_{2}}
$$

respectively. Intensity $\rho_{1}$ and $\rho_{2}$ can be interpreted as expected number of arrivals per mean service time. The condition for stability of the system is both $\rho_{1}, \rho_{2}$ are less unity.

Basic properties of queueing networks are introduced in Disney [1]. Burke [2], Beautler and Melamed [3] showed that the input process to a service center in a network with feedback is not Poisson in general. It is for this reason that Jacksons result is remarkable. Jacksons [4] theorem states that each node behaves like an independent queue.

The product form solution to open network of Markovian queues with feedback is also discussed in Jackson [4]. Simon and Foley [5], Melamed [6] pointed out that computation of response time distribution is difficult even for Jacksonian networks without feedback. Disney and Kiessel [7] discussed traffic process in queueing networks thorough Markov renewal approach. Thiruvaiyaru, Basawa and Bhat [8] established Maximum likelihood estimators of the parameters of an open Jackson network are derived, and their joint asymptotic normality. The problem of estimation for tandem queues is discussed as a special case of the Jackson system. These results are valid when the system is not necessarily in equilibrium. Thiruvaiyaru and Basawa [9] considered the problem of estimation for the parameters in a Jackson's type queueing network with the arrival at each node following renewal process and service time distribution being arbitrary. Open queueing networks are useful in studying the behavior of computer communication networks (Kleinrock [10]). More approach to queueing network analysis 


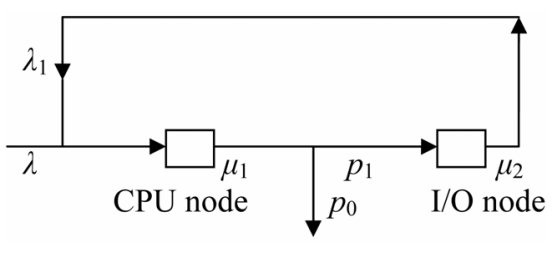

Figure 1. An open queueing network with feedback.

developed by Buzen and Denning [11]. Efron [12-14] the greatest statistician in the field of nonparametric resampling approach, originally developed and proposed the bootstrap, which is a resampling technique that can be effectively applied to estimate the sampling distribution of any statistic. Specifically, one can utilize the bootstrap method to approximate the sampling distribution of a statistic defined by a random sample from a population with unknown probability distribution. And due to the popularity of PC and statistical software, today the bootstrap becomes the most powerful nonparametric estimation procedure. Based upon the bootstrap resampling technique, most statisticians utilize the standard bootstrap (SB), percentile bootstrap (PB), and bias-corrected and accelerated bootstrap $(\mathrm{BCaB})$ approaches to produce confidence intervals for practical problems.

Besides the standard bootstrap (SB) technique, Rubin [15] presented the Bayesian bootstrap (BB) technique of resampling. Miller [16] showed that the SB can be regarded as an extension of the jackknife. The $\mathrm{BB}$ is a natural Bayesian analogue of the SB. The BB simulates the posterior distribution of parameters under particular model specifications, whereas the SB simulates the estimated sampling distribution of a statistic estimating the parameters. Both SB and BB can be applied to construct confidence intervals of intensity for a queueing system with distribution-free inter-arrival and service times.

Chu and Ke [17] constructed new confidence intervals of mean response time for an M/G/1 FCFS queueing system. Also, they performed the accuracy of bootstrap confidence intervals through calculating the coverage probability and the average length of confidence intervals. Chu and Ke [18] proposed a consistent and asymptotically normal (CAN) estimator of the mean response time for a $\mathrm{G} / \mathrm{M} / 1$ queueing system, which is based on the fixed point of empirical Laplace function. Ke and Chu [19] proposed a consistent and asymptotically normal estimator of intensity for a queueing system with distribution-free interarrival and service times. Also, they computed confidence intervals, testing statistical hypothesis of intensity and power function associated with it in this paper. Ke and Chu [20] constructed new confidence intervals of intensity for a queueing system, which are based on different bootstrap methods. They also performed the accuracy of these bootstrap confidence intervals through calculating the coverage probability and the expected length of confidence intervals. They first proposed bootstrapping technique and concept of relative coverage to queueing system. They studied five estimation approaches of intensity for a queueing system with distribution free inter-arrival and service times for short run. They have introduced a new measure called relative coverage to assess the efficient performances of confidence intervals.

In this paper we propose non parametric interval estimation approach to intensities $\rho_{1}, \rho_{2}$ for a open queueing network with feedback. In Section 2 we prove that the natural estimators $\hat{\rho}_{1}, \hat{\rho}_{2}$ of intensities $\rho_{1}$ and $\rho_{2}$ are strongly consistent and asymptotically normal (CAN). Based on the asymptotical normality of $\hat{\rho}_{1}, \hat{\rho}_{2}$, we can construct a CAN confidence interval of $\rho_{1}$ and $\rho_{2}$. Next in Section 3 we establish the SB confidence interval of $\rho_{1}, \rho_{2}$ via the standard bootstrap approach. In Section 4 we developed the derivation of the BB confidence interval of $\rho_{1}, \rho_{2}$ in terms of the Bayesian bootstrap approach. The percentile bootstrap (PB) confidence intervals of $\rho_{1}, \rho_{2}$ are developed in Section 5. In Section 6 we developed the bias-corrected and accelerated bootstrap (BCaB) confidence intervals. A numerical simulation study is conducted in Section 7 to demonstrate performances of the interval estimation approaches for an open queueing network with feedback using short run data. All simulation results are shown by appropriate tables for illustrating performances of the five interval estimation approaches. Finally, we make some conclusions in Section 8.

\section{Nonparametric Statistical Inference of Intensities}

Let $X_{1}$ and $Y_{1}$ be nonnegative random variables represents the inter-arrival and service time at CPU node. Similarly $X_{2}$ and $Y_{2}$ be nonnegative random variables represents the inter-arrival and service time at I/O node respectively. Given that a job just completed CPU node burst, it will next request I/O node service with probability $p_{1}$ and with probability $p_{0}$, where $p_{0}=1-p_{1}$ departs from the system. The random variables at CPU node and I/O node are independent. The intensities are defined as follows:

$$
\rho_{1}=\frac{p_{0} \mu_{Y_{1}}}{\mu_{X_{1}}} \text { and } \rho_{2}=\frac{p_{0} \mu_{Y_{2}}}{p_{1} \mu_{X_{2}}},
$$

where $\mu_{X_{1}}, \mu_{X_{2}}$ denote the mean inter-arrival times at CPU node and I/O node respectively. Similarly $\mu_{Y_{1}}, \mu_{Y_{2}}$ denote the mean service times at CPU node and I/O node respectively. Equation (2) is equivalent to Equation (1).

\subsection{Estimating Intensities}

Assume that $X_{11}, X_{12}, \cdots, X_{1 n}$ is a random sample drawn 
from $X_{1}$ and $p_{0} Y_{11}, p_{0} Y_{12}, \cdots, p_{0} Y_{1 n}$ is a random sample drawn from $Y_{1}$. Let $\left(X_{1 i}, p_{0} Y_{1 i}\right)$ represents inter-arrival time and service time for the $i^{\text {th }}$ customer of CPU node. Similarly assume $p_{1} X_{21}, p_{1} X_{22}, \cdots, p_{1} X_{2 m}$ is a random sample drawn from $X_{2}$ and $p_{0} Y_{21}, p_{0} Y_{22}, \cdots, p_{0} Y_{2 m}$ is a random sample drawn from $Y_{2}$. Let $\left(p_{1} X_{2 i}, p_{0} Y_{2 i}\right)$ represents inter-arrival time and service time for the $i^{\text {th }}$ customer of I/O node.

Let $\bar{X}_{1}, \bar{X}_{2}, \bar{Y}_{1}, \bar{Y}_{2}$ be the sample means of $X_{1}, X_{2}, Y_{1}$, $Y_{2}$ respectively.

$$
\begin{aligned}
& \bar{X}_{1}=\frac{1}{n} \sum_{i=1}^{n} X_{1 i}, \bar{X}_{2}=\frac{1}{m} \sum_{i=1}^{m} p_{1} X_{2 i} \\
& \bar{Y}_{1}=\frac{1}{n} \sum_{i=1}^{n} p_{0} Y_{1 i}, \bar{Y}_{2}=\frac{1}{m} \sum_{i=1}^{m} p_{0} Y_{2 i}
\end{aligned}
$$

According to the Strong Law of Large Numbers, we know that $\bar{X}_{1}, \bar{X}_{2}, \bar{Y}_{1}, \bar{Y}_{2}$ are strongly consistent estimator of $\mu_{X_{1}}, \mu_{X_{2}}, \mu_{Y_{1}}, \mu_{Y_{2}}$ respectively. Thus a strongly consistent estimator of intensities are given by

$$
\hat{\rho}_{1}=\frac{\bar{Y}_{1}}{\bar{X}_{1}}, \hat{\rho}_{2}=\frac{\bar{Y}_{2}}{\bar{X}_{2}}
$$

In practical queueing network, the true distributions of $X_{1}, X_{2}, Y_{1}, Y_{2}$ are rarely known, so the exact distributions of $\hat{\rho}_{1}, \hat{\rho}_{2}$ cannot be derived. But under the assumption that $X_{1}$ and $Y_{1}, X_{2}$ and $Y_{2}$ being independent, the asymptotic distributions of $\hat{\rho}_{1}, \hat{\rho}_{2}$ can be developed by the following procedures.

Firstly, according to the Central Limit Theorem (see [21] p. 234), we have

$$
\begin{aligned}
& \sqrt{n}\left(\bar{X}_{1}-\mu_{X_{1}}\right) \stackrel{D}{\longrightarrow} N\left(0, \sigma_{X_{1}}^{2}\right) \text { and } \\
& \sqrt{n}\left(\bar{Y}_{1}-p_{0} \mu_{Y_{1}}\right) \stackrel{D}{\longrightarrow} N\left(0, \sigma_{Y_{1}}^{2}\right)
\end{aligned}
$$

where $\sigma_{X_{1}}^{2}$ and $\sigma_{Y_{1}}^{2}$ are variances of $X_{1}$ and $Y_{1}$, respectively.

Also,

$$
\begin{aligned}
& \sqrt{m}\left(\bar{X}_{2}-p_{1} \mu_{X_{2}}\right) \stackrel{D}{\longrightarrow} N\left(0, \sigma_{X_{2}}^{2}\right) \text { and } \\
& \sqrt{m}\left(\bar{Y}_{2}-p_{0} \mu_{Y_{2}}\right) \stackrel{D}{\longrightarrow} N\left(0, \sigma_{Y_{2}}^{2}\right),
\end{aligned}
$$

where $\sigma_{X_{2}}^{2}$ and $\sigma_{Y_{2}}^{2}$ are variances of $X_{2}$ and $Y_{2}$, respectively, and $\stackrel{D}{\longrightarrow}$ denotes convergence in distribution.

Next note that

$$
\begin{aligned}
& \sqrt{n}\left(\hat{\rho}_{1}-\rho_{1}\right) \\
& =\sqrt{n}\left(\frac{\bar{Y}_{1}}{\bar{X}_{1}}-\frac{p_{0} \mu_{Y_{1}}}{\mu_{X_{1}}}\right) \\
& =\frac{\sqrt{n}\left[\mu_{X_{1}}\left(\bar{Y}_{1}-p_{0} \mu_{Y_{1}}\right)-p_{0} \mu_{Y_{1}}\left(\bar{X}_{1}-\mu_{X_{1}}\right)\right]}{\mu_{X_{1}} \bar{X}_{1}} .
\end{aligned}
$$

Also,

$$
\begin{aligned}
& \sqrt{m}\left(\hat{\rho}_{2}-\rho_{2}\right) \\
& =\sqrt{m}\left(\frac{\bar{Y}_{2}}{\bar{X}_{2}}-\frac{p_{0} \mu_{Y_{2}}}{p_{1} \mu_{X_{2}}}\right) \\
& =\frac{\sqrt{m}\left[p_{1} \mu_{X_{2}}\left(\bar{Y}_{2}-p_{0} \mu_{Y_{2}}\right)-p_{0} \mu_{Y_{2}}\left(\bar{X}_{2}-p_{1} \mu_{X_{2}}\right)\right]}{p_{1} \mu_{X_{2}} \bar{X}_{2}} .
\end{aligned}
$$

Therefore by the Slutsky's theorem [see [21] p. 227], we get

$$
\sqrt{n}\left(\hat{\rho}_{1}-\rho_{1}\right) \stackrel{D}{\longrightarrow} N\left(0, \sigma_{1}^{2}\right)
$$

where $\sigma_{1}^{2}=\frac{\mu_{X_{1}}^{2} \sigma_{Y_{1}}^{2}+p_{0}^{2} \mu_{Y_{1}}^{2} \sigma_{X_{1}}^{2}}{\mu_{X_{1}}^{4}}$.

And

$$
\sqrt{m}\left(\hat{\rho}_{2}-\rho_{2}\right) \stackrel{D}{\longrightarrow} N\left(0, \sigma_{2}^{2}\right)
$$

where $\sigma_{2}^{2}=\frac{p_{1}^{2} \mu_{X_{2}}^{2} \sigma_{Y_{2}}^{2}+p_{0}^{2} \mu_{Y_{2}}^{2} \sigma_{X_{2}}^{2}}{p_{1}^{2} \mu_{X_{2}}^{4}}$.

Now, set

$$
\begin{aligned}
& \hat{\sigma}_{1}^{2}=\frac{\bar{X}_{1}^{2} S_{Y_{1}}^{2}+p_{0}^{2} \bar{Y}_{1}^{2} S_{X_{1}}^{2}}{\bar{X}_{1}^{4}} \text { and } \\
& \hat{\sigma}_{2}^{2}=\frac{p_{1}^{2} \bar{X}_{2}^{2} S_{Y_{2}}^{2}+p_{0}^{2} \bar{Y}_{2}^{2} S_{X_{2}}^{2}}{p_{1}^{2} \bar{X}_{2}^{4}},
\end{aligned}
$$

where

$$
\begin{aligned}
& S_{X_{1}}^{2}=\frac{1}{n} \sum_{i=1}^{n}\left(X_{1 i}-\bar{X}_{1}\right)^{2}, S_{Y_{1}}^{2}=\frac{1}{n} \sum_{i=1}^{n}\left(p_{0} Y_{1 i}-\bar{Y}_{1}\right)^{2} \\
& S_{X_{2}}^{2}=\frac{1}{m} \sum_{i=1}^{m}\left(p_{1} X_{2 i}-\bar{X}_{2}\right)^{2} \text { and } S_{Y_{2}}^{2}=\frac{1}{m} \sum_{i=1}^{m}\left(p_{0} Y_{2 i}-\bar{Y}_{2}\right)^{2} .
\end{aligned}
$$

Then $\hat{\sigma}_{1}^{2}, \hat{\sigma}_{2}^{2}$ are strongly consistent estimators of $\sigma_{1}^{2}, \sigma_{2}^{2}$ respectively. Applying the Slutsky's theorem once again, we deduce that

$$
\begin{aligned}
& \frac{\sqrt{n}\left(\hat{\rho}_{1}-\rho_{1}\right)}{\hat{\sigma}_{1}} \stackrel{D}{\longrightarrow} N(0,1) \text { and } \\
& \frac{\sqrt{m}\left(\hat{\rho}_{2}-\rho_{2}\right)}{\hat{\sigma}_{2}} \stackrel{D}{\longrightarrow} N(0,1)
\end{aligned}
$$

Thus $\hat{\rho}_{1}, \hat{\rho}_{2}$ are strongly consistent and asymptotically normal (CAN) estimators with approximate variances $\frac{\hat{\sigma}_{1}^{2}}{n}, \frac{\hat{\sigma}_{2}^{2}}{m}$ respectively.

\subsection{Confidence Intervals}

Using the CAN estimators $\hat{\rho}_{1}, \hat{\rho}_{2}$ and its associated approximate variances $\frac{\hat{\sigma}_{1}^{2}}{n}, \frac{\hat{\sigma}_{2}^{2}}{m}$ we construct a confi- 
dence intervals of intensities $\rho_{1} \& \rho_{2}$ for a open queueing network with feedback. Let $z_{\alpha}$ be the upper $\alpha^{\text {th }}$ quantile of the standard normal distribution, by the asymptotic distribution of $\frac{\sqrt{n}\left(\hat{\rho}_{1}-\rho_{1}\right)}{\hat{\sigma}_{1}} \& \frac{\sqrt{m}\left(\hat{\rho}_{2}-\rho_{2}\right)}{\hat{\sigma}_{2}}$ in expression (9), an approximate $100(1-\alpha) \%$ confidence interval of $\rho_{1} \& \rho_{2}$ are obtained as

$$
\begin{aligned}
1-\alpha & \approx P\left(-z_{\alpha / 2} \leq \frac{\sqrt{n}\left(\hat{\rho}_{1}-\rho_{1}\right)}{\hat{\sigma}_{1}} \leq z_{\alpha / 2}\right) \\
& =P\left(\hat{\rho}_{1}-\frac{z_{\alpha / 2} \hat{\sigma}_{1}}{\sqrt{n}} \leq \rho_{1} \leq \hat{\rho}_{1}+\frac{z_{\alpha / 2} \hat{\sigma}_{1}}{\sqrt{n}}\right)
\end{aligned}
$$

Consequently, an approximate $100(1-\alpha) \%$ confidence interval of $\rho_{1}$ is

$$
\left(\hat{\rho}_{1}-\frac{z_{\alpha / 2} \hat{\sigma}_{1}}{\sqrt{n}}, \hat{\rho}_{1}+\frac{z_{\alpha / 2} \hat{\sigma}_{1}}{\sqrt{n}}\right) .
$$

Similarly, an approximate $100(1-\alpha) \%$ confidence interval of $\rho_{2}$ is

$$
\left(\hat{\rho}_{2}-\frac{z_{\alpha / 2} \hat{\sigma}_{2}}{\sqrt{m}}, \hat{\rho}_{2}+\frac{z_{\alpha / 2} \hat{\sigma}_{2}}{\sqrt{m}}\right) .
$$

\section{Standard Bootstrap Confidence Intervals of Intensities}

Now the bootstrap confidence intervals are developed as follows:

Let $x_{11}, x_{12}, \cdots, x_{1 n}$ be a random sample of $\mathrm{n}$ observations taken from the population $X_{1}$ and $p_{0} y_{11}, p_{0} y_{12}, \cdots$, $p_{0} y_{1 n}$ be a random sample of $n$ observations taken from the population $Y_{1}$. According to the bootstrap procedure, a simple random sample $x_{11}^{*}, x_{12}^{*}, \cdots, x_{1 n}^{*}$ can be taken from the empirical distribution function of $x_{11}, x_{12}, \cdots, x_{1 n}$ called a bootstrap sample from $x_{11}, x_{12}, \cdots, x_{1 n}$. Also, we can draw a bootstrap sample $p_{0} y_{11}^{*}, p_{0} y_{12}^{*}, \cdots, p_{0} y_{1 n}^{*}$ from $p_{0} y_{11}, p_{0} y_{12}, \cdots, p_{0} y_{1 n}$. It follows from Equation (2) that an estimate of intensity $\rho_{1}$ can be calculated from bootstrap samples as

$$
\hat{\rho}_{1}^{*}=\frac{\bar{y}_{1}^{*}}{\bar{x}_{1}^{*}},
$$

where $\bar{x}_{1}^{*}$ and $\bar{y}_{1}^{*}$ are the sample means of $x_{11}^{*}, x_{12}^{*}, \cdots$, $x_{1 n}^{*}$ and $p_{0} y_{11}^{*}, p_{0} y_{12}^{*}, \cdots, p_{0} y_{1 n}^{*}$ respectively and $\hat{\rho}_{1}^{*}$ is called a bootstrap estimate of $\rho_{1}$. The above resampling process can be repeated $N_{1}$ times. The $N_{1}$ bootstrap estimates $\hat{\rho}_{11}^{*}, \hat{\rho}_{12}^{*}, \cdots, \hat{\rho}_{1 N_{1}}^{*}$ can be computed from the bootstrap resamples. Averaging the $N_{1}$ bootstrap estimates we obtain that

$$
\hat{\rho}_{N_{1}}=\frac{1}{N_{1}} \sum_{i=1}^{N_{1}} \hat{\rho}_{1 i}^{*}
$$

is the bootstrap estimate of $\rho_{1}$. And the standard devia- tion of $\hat{\rho}_{1}$ can be estimated by

$$
s d\left(\hat{\rho}_{N_{1}}\right)=\left\{\frac{1}{N_{1}-1} \sum_{i=1}^{N_{1}}\left(\hat{\rho}_{1 i}^{*}-\hat{\rho}_{N_{1}}\right)^{2}\right\}^{1 / 2} .
$$

Because the central limit theorem implies that the distribution of $\hat{\rho}_{1}$ is approximately normal. A $100(1-\alpha) \%$ SB confidence interval for $\rho_{1}$ is

$$
\left(\hat{\rho}_{1}-z_{\alpha / 2} s d\left(\hat{\rho}_{N_{1}}\right), \hat{\rho}_{1}+z_{\alpha / 2} s d\left(\hat{\rho}_{N_{1}}\right)\right),
$$

Similarly $p_{1} x_{21}, p_{1} x_{22}, \cdots, p_{1} x_{2 m}$ is a random sample of $m$ observation drawn from population $X_{2}$ and $p_{0} y_{21}$, $p_{0} y_{22}, \cdots, p_{0} y_{2 m}$ is a sample of $\mathrm{m}$ observations taken from the population $Y_{2}$. According to the bootstrap procedure, a simple random sample $p_{1} x_{21}^{*}, p_{1} x_{22}^{*}, \cdots, p_{1} x_{2 m}^{*}$ can be taken from the empirical distribution function of $p_{1} x_{21}, p_{1} x_{22}, \cdots, p_{1} x_{2 m}$ called a bootstrap sample from $p_{1} x_{21}, p_{1} x_{22}, \cdots, p_{1} x_{2 m}$. Also, we can draw a bootstrap sample $p_{0} y_{21}^{*}, p_{0} y_{22}^{*}, \cdots, p_{0} y_{2 m}^{*}$ from $p_{0} y_{21}, p_{0} y_{22}, \cdots$, $p_{0} y_{2 m}$. An estimate of intensity $\rho_{2}$ can be calculated from bootstrap samples as

$$
\hat{\rho}_{2}^{*}=\frac{\bar{y}_{2}^{*}}{\bar{x}_{2}^{*}},
$$

where $\bar{x}_{2}^{*}$ and $\bar{y}_{2}^{*}$ are the sample means of $p_{1} x_{21}^{*}$, $p_{1} x_{22}^{*}, \cdots, p_{1} x_{2 m}^{*} \quad$ and $p_{0} y_{21}^{*}, p_{0} y_{22}^{*}, \cdots, p_{0} y_{2 m}^{*} \quad$ respectively and $\hat{\rho}_{2}^{*}$ is called a bootstrap estimate of $\rho_{2}$. The above resampling process can be repeated $M_{1}$ times. The $M_{1}$ bootstrap estimates $\hat{\rho}_{21}^{*}, \hat{\rho}_{22}^{*}, \cdots, \hat{\rho}_{2 M_{1}}^{*}$ can be computed from the bootstrap resamples. Averaging the $M_{1}$ bootstrap estimates we obtain that

$$
\hat{\rho}_{M_{1}}=\frac{1}{M_{1}} \sum_{i=1}^{M_{1}} \hat{\rho}_{2 i}^{*}
$$

is the bootstrap estimate of $\rho_{2}$. And the standard deviation of $\hat{\rho}_{2}$ can be estimated by

$$
s d\left(\hat{\rho}_{M_{1}}\right)=\left\{\frac{1}{M_{1}-1} \sum_{i=1}^{M}\left(\hat{\rho}_{2 i}^{*}-\hat{\rho}_{M_{1}}\right)^{2}\right\}^{1 / 2} .
$$

Because the central limit theorem implies that the distribution of $\hat{\rho}_{2}$ is approximately normal. A $100(1-\alpha) \%$ SB confidence interval for $\rho_{2}$ is

$$
\left(\hat{\rho}_{2}-z_{\alpha / 2} s d\left(\hat{\rho}_{M_{1}}\right), \hat{\rho}_{2}+z_{\alpha / 2} s d\left(\hat{\rho}_{M_{1}}\right)\right),
$$

\section{Bayesian Bootstrap Confidence Intervals of Intensities}

The Bayesian bootstrap is analogous to the standard bootstrap. Each BB replication generates a posterior probability for each $x_{1 i}$. Specifically, one BB replication is generated by drawing $n-1$ uniform $(0,1)$ random numbers $r_{1}, r_{2}, \cdots, r_{n-1}$, ordering them, and calculating the gaps $w_{1 i}=r_{(i)}-r_{(i-1)}, i=1,2, \cdots, n$, where $r_{(0)}=0$ and 
$r_{(n)}=1$. Then $w_{1 i}=\left(w_{11}, w_{12}, \cdots, w_{1 n}\right)$ is the vector of probabilities attached to the inter-arrival data values $x_{11}, x_{12}, \cdots, x_{1 n}$ in that $\mathrm{BB}$ replication. Considering all $\mathrm{BB}$ replications gives the $\mathrm{BB}$ distribution of the distribution of $X_{1}$ and thus of any parameter of this distribution. Hence for $\mu_{X_{1}}$ (the mean of $X_{1}$ ), in each BB replication we calculate $\mu_{X_{1}}$ as if $w_{1 i}$ were the probability that $X_{1}=x_{i}$ that is, we calculate $\bar{x}_{1}^{* *}=\sum_{i=1}^{n} w_{1 i} x_{1 i}$. The distribution of the values of $\bar{x}_{1}^{* *}$ overall BB replications is the BB distribution of $\mu_{X_{1}}$.

Also, generating a vector of probabilities $v_{1}=\left(v_{11}, v_{12}, \cdots, v_{1 n}\right)$ attached to the service time data values $p_{0} y_{11}, p_{0} y_{12}, \cdots, p_{0} y_{1 n}$ in a BB replication, and we calculate $\bar{y}_{1}^{* *}=p_{0} \sum_{i=1}^{n} v_{1 i} y_{1 i}$ for $\mu_{Y_{1}}$ (the mean of $Y_{1}$ ). Then in terms of equation (2) an estimate of intensity $\rho_{1}$ can be calculated from BB replications as

$$
\hat{\rho}_{1}^{* *}=\frac{\bar{y}_{1}^{* *}}{\bar{x}_{1}^{* *}},
$$

where $\hat{\rho}_{1}^{* *}$ is called a Bayesian bootstrap estimate of $\rho_{1}$. The above BB process can be repeated $N_{1}$ times. The $N_{1}$ BB estimates, $\hat{\rho}_{11}^{* *}, \hat{\rho}_{12}^{* *}, \cdots, \hat{\rho}_{1 N_{1}}^{* *}$ can be computed from the BB replications. Averaging the $N_{1}$ BB estimates, we obtain that

$$
\hat{\rho}_{B B}^{\prime}=\frac{1}{N_{1}} \sum_{j=1}^{N_{1}} \hat{\rho}_{1 j}^{* *},
$$

is the BB estimate of $\rho_{1}$. And the standard deviation of $\hat{\rho}_{1}$ can be estimated by

$$
s d\left(\hat{\rho}_{B B}^{\prime}\right)=\left\{\frac{1}{N_{1}-1} \sum_{j=1}^{N_{1}}\left(\hat{\rho}_{1 j}^{* *}-\hat{\rho}_{\mathrm{BB}}^{\prime}\right)^{2}\right\}^{\frac{1}{2}} .
$$

Applying the asymptotical normality of $\hat{\rho}_{1}$, a $100(1-\alpha) \%$ BB confidence interval for $\rho_{1}$ is

$$
\left(\hat{\rho}_{1}-z_{\alpha / 2} s d\left(\hat{\rho}_{B B}^{\prime}\right), \hat{\rho}_{1}+z_{\alpha / 2} s d\left(\hat{\rho}_{B B}^{\prime}\right)\right) .
$$

Similarly each $\mathrm{BB}$ replication generates a posterior probability for each $x_{2 i}$. Specifically, one BB replication is generated by drawing $m-1$ uniform $(0,1)$ random numbers $r_{1}, r_{2}, \cdots, r_{m-1}$, ordering them and calculating the gaps $w_{2 i}=r_{(i)}-r_{(i-1)}, i=1,2, \cdots, m$, where $r_{(0)}=0$ and $r_{(m)}=1$. Then $w_{2 i}=\left(w_{21}, w_{22}, \cdots, w_{2 m}\right)$ is the vector of probabilities attached to the inter-arrival data values $p_{1} x_{21}, p_{1} x_{22}, \cdots, p_{1} x_{2 m}$ in that BB replication. Considering all $\mathrm{BB}$ replications gives the $\mathrm{BB}$ distribution of the distribution of $X_{2}$ and thus of any parameter of this distribution. Hence for $\mu_{X_{2}}$ (the mean of $X_{2}$ ), in each BB replication we calculate $\mu_{X_{2}}$ as if $w_{2 i}$ were the probability that $X_{2}=x_{2 i}$ that is, we calculate $\bar{x}_{2}^{* *}=p_{1} \sum_{i=1}^{m} w_{2 i} x_{2 i}$. The distribution of the values of $\bar{x}_{2}^{* *}$ over all BB replications is the BB distribution of $\mu_{X_{2}}$.

Also, generating a vector of probabilities

$v_{2}=\left(v_{21}, v_{22}, \cdots, v_{2 m}\right)$ attached to the service time data values $p_{0} y_{21}, p_{0} y_{22}, \cdots, p_{0} y_{2 m}$ in a BB replication, and we calculate $\bar{y}_{2}^{* *}=p_{0} \sum_{i=1}^{m} v_{2 i} y_{2 i}$ for $\mu_{Y_{2}}$ (the mean of $Y_{2}$ ).

Then in terms of Equation (2) an estimate of intensity $\rho_{2}$ can be calculated from BB replications as

$$
\hat{\rho}_{2}^{* *}=\frac{\bar{y}_{2}^{* *}}{\bar{x}_{2}^{* *}},
$$

where $\hat{\rho}_{2}^{* *}$ is called a Bayesian bootstrap estimate of $\rho_{2}$. The above BB process can be repeated $M_{1}$ times. The $M_{1} \mathrm{BB}$ estimates, $\hat{\rho}_{21}^{* *}, \hat{\rho}_{22}^{* *}, \cdots, \hat{\rho}_{2 M_{1}}^{* *}$ can be computed from the $\mathrm{BB}$ replications. Averaging the $M_{1} \mathrm{BB}$ estimates, we obtain that

$$
\hat{\rho}_{B B}^{\prime \prime}=\frac{1}{M_{1}} \sum_{j=1}^{M_{1}} \hat{\rho}_{2 j}^{* *},
$$

is the $B B$ estimate of $\rho_{2}$. And the standard deviation of $\hat{\rho}_{2}$ can be estimated by

$$
s d\left(\hat{\rho}_{B B}^{\prime \prime}\right)=\left\{\frac{1}{M_{1}-1} \sum_{j=1}^{M_{1}}\left(\hat{\rho}_{2 j}^{* *}-\hat{\rho}_{B B}^{\prime \prime}\right)^{2}\right\} .
$$

Applying the asymptotical normality of $\hat{\rho}_{2}$, a $100(1-\alpha) \%$ BB confidence interval for $\rho_{2}$ is

$$
\left(\hat{\rho}_{2}-z_{\alpha / 2} s d\left(\hat{\rho}_{B B}^{\prime \prime}\right), \hat{\rho}_{2}+z_{\alpha / 2} s d\left(\hat{\rho}_{B B}^{\prime \prime}\right)\right)
$$

\section{Percentile Bootstrap Confidence Intervals of Intensities}

Let $\hat{\rho}_{11}^{*}, \hat{\rho}_{12}^{*}, \cdots, \hat{\rho}_{1 N_{1}}^{*}$ and $\hat{\rho}_{21}^{*}, \hat{\rho}_{22}^{*}, \cdots, \hat{\rho}_{2 M_{1}}^{*}$ call the bootstrap distribution of $\hat{\rho}_{1}, \hat{\rho}_{2}$ respectively. Let $\hat{\rho}_{1}^{*}(1)$, $\hat{\rho}_{1}^{*}(2), \cdots, \hat{\rho}_{1}^{*}\left(N_{1}\right)$ and $\hat{\rho}_{2}^{*}(1), \hat{\rho}_{2}^{*}(2), \cdots, \hat{\rho}_{2}^{*}\left(M_{1}\right)$ be order statistics of $\hat{\rho}_{11}^{*}, \hat{\rho}_{12}^{*}, \cdots, \hat{\rho}_{1 N_{1}}^{*}$ and $\hat{\rho}_{21}^{*}, \hat{\rho}_{22}^{*}, \cdots, \hat{\rho}_{2 M_{1}}^{*}$ respectively. Then utilizing the $100(\alpha / 2)$ th and $100(1-\alpha / 2)$ th percentage points of the bootstrap distribution, a $100(1-\alpha) \% \mathrm{~PB}$ confidence interval for $\rho_{1}$, $\rho_{2}$ are obtained as

$$
\begin{aligned}
& \left(\hat{\rho}_{1}^{*}\left(\left[N_{1}\left(\frac{\alpha}{2}\right)\right]\right), \hat{\rho}_{1}^{*}\left(\left[N_{1}\left(1-\frac{\alpha}{2}\right)\right]\right)\right), \\
& \left(\hat{\rho}_{2}^{*}\left(\left[M_{1}\left(\frac{\alpha}{2}\right)\right]\right), \hat{\rho}_{2}^{*}\left(\left[M_{1}\left(1-\frac{\alpha}{2}\right)\right]\right)\right),
\end{aligned}
$$

where $[x]$ denotes the greatest integer less than or equal to $x$.

\section{Bias-Corrected and Accelerated Bootstrap Confidence Intervals of Intensities}

The bootstrap distribution $\hat{\rho}_{11}^{*}, \hat{\rho}_{12}^{*}, \cdots, \hat{\rho}_{1 N_{1}}^{*}$ and $\hat{\rho}_{21}^{*}$, 
$\hat{\rho}_{22}^{*}, \cdots, \hat{\rho}_{2 M_{1}}^{*}$ may be biased. This method is designed to correct this potential bias of the bootstrap designed. Set

$$
p^{\prime}=\sum_{j=1}^{N_{1}} \frac{I\left(\hat{\rho}_{1 j}^{*}<\hat{\rho}_{1}\right)}{N_{1}} \text { and } p^{\prime \prime}=\sum_{j=1}^{M_{1}} \frac{I\left(\hat{\rho}_{2 j}^{*}<\hat{\rho}_{2}\right)}{M_{1}},
$$

where $I(\cdot)$ is the indicator function. Define $\hat{z}_{0}=\phi^{-1}\left(p^{\prime}\right)$ and $\hat{z}_{1}=\phi^{-1}\left(p^{\prime \prime}\right)$, where $\phi^{-1}$ denotes the inverse function of the standard normal distribution $\phi$. Except for correcting the potential bias of the bootstrap distribution, we can accelerate convergence of bootstrap distribution. Let $\tilde{X}_{1}(i)$ and $p_{0} \tilde{Y}_{1}(i)$ denote the original samples with the $i^{\text {th }}$ observation $x_{1 i}$ and $p_{0} y_{1 i}$ deleted, also let $\hat{\rho}_{1 i}$ be the estimator of $\rho_{1}$ calculated by using $\tilde{X}_{1}(i)$ and $p_{0} \tilde{Y}_{1}(i)$ Define $\tilde{\rho}_{1}=\frac{1}{n} \sum_{i=1}^{n} \hat{\rho}_{1 i}$, Similarly $p_{1} \tilde{X}_{2}(i)$, $p_{0} \tilde{Y}_{2}(i)$ denote the original samples with the $i^{\text {th }}$ observation $p_{1} x_{2 i}$ and $p_{0} y_{2 i}$ deleted, also $\hat{\rho}_{2 i}$ be the estimator of $\rho_{2}$ calculated by using $p_{1} \tilde{X}_{2}(i)$ and $p_{0} \tilde{Y}_{2}(i)$.

Define $\tilde{\rho}_{2}=\frac{1}{m} \sum_{i=1}^{m} \hat{\rho}_{2 i}$

And

$$
\begin{aligned}
& \hat{a}_{1}=\frac{\sum_{i=1}^{n}\left(\tilde{\rho}_{1}-\hat{\rho}_{1 i}\right)^{3}}{\left\{6\left(\sum_{i=1}^{n}\left(\tilde{\rho}_{1}-\hat{\rho}_{1 i}\right)^{2}\right)^{\left(\frac{3}{2}\right)}\right\}}, \\
& \hat{a}_{2}=\frac{\sum_{i=1}^{m}\left(\tilde{\rho}_{2}-\hat{\rho}_{2 i}\right)^{3}}{\left\{6\left(\sum_{i=1}^{m}\left(\tilde{\rho}_{2}-\hat{\rho}_{2 i}\right)^{2}\right)^{\left(\frac{3}{2}\right)}\right\}}
\end{aligned}
$$

where $\hat{z}_{0}, \hat{z}_{1}, \hat{a}_{1}$, and $\hat{a}_{2}$ are named bias-correction and acceleration respectively.

Thus a $100(1-\alpha) \%$ Bias-corrected and accelerated bootstrap (BCaB) Confidence Interval of intensities $\rho_{1}$, $\rho_{2}$ are constructed by

$$
\begin{aligned}
& \left(\hat{\rho}_{1}^{*}\left(\left[N_{1} \alpha_{1}\right]\right), \hat{\rho}_{1}^{*}\left(\left[N_{1} \alpha_{2}\right]\right)\right) \\
& \left(\hat{\rho}_{2}^{*}\left(\left[M_{1} \alpha_{3}\right]\right), \hat{\rho}_{2}^{*}\left(\left[M_{1} \alpha_{4}\right]\right)\right)
\end{aligned}
$$

where

$$
\begin{aligned}
& \alpha_{1}=\phi\left\{\hat{z}_{0}+\frac{\left(\hat{z}_{0}-z_{\alpha / 2}\right)}{1-\hat{a}_{1}\left(\hat{z}_{0}-z_{\alpha / 2}\right)}\right\} \\
& \alpha_{2}=\phi\left\{\hat{z}_{0}+\frac{\left(\hat{z}_{0}+z_{\alpha / 2}\right)}{1-\hat{a}_{1}\left(\hat{z}_{0}+z_{\alpha / 2}\right)}\right\}
\end{aligned}
$$

$$
\begin{aligned}
& \alpha_{3}=\phi\left\{\hat{z}_{1}+\frac{\left(\hat{z}_{1}-z_{\alpha / 2}\right)}{1-\hat{a}_{2}\left(\hat{z}_{1}-z_{\alpha / 2}\right)}\right\} \\
& \alpha_{4}=\phi\left\{\hat{z}_{1}+\frac{\left(\hat{z}_{1}+z_{\alpha / 2}\right)}{1-\hat{a}_{2}\left(\hat{z}_{1}+z_{\alpha / 2}\right)}\right\}
\end{aligned}
$$

\section{Simulation Study}

To evaluate performances of the different interval estimation approaches mentioned above for an open queueing network with feedback using short run data, a numerical simulation study was undertaken. Most of the statisticians assess performances of interval estimations in terms of coverage percentages or average lengths of confidence intervals. However, through simulation study in the research work, we find that larger coverage percentages of confidence interval may often be due to wider standard deviation of interval estimation methods. Moreover, narrower confidence intervals may often lead to smaller coverage percentages. Hence, both coverage percentage and average length are not efficient for appraising interval estimation methods. In order to overcome above two shortcomings, we propose a measure called relative coverage to evaluate performances of interval estimation methods where,

$$
\text { Relative coverage }=\frac{\text { Coverage percentage }}{\text { Average length }} \text {. }
$$

The larger of the relative coverage implies the better performance of the corresponding confidence interval. In order to reach this goal, we set a continuous distribution with mean $1 / \lambda$ on inter-arrival time $X_{1}$ and $X_{2}$. Also set continuous distribution with mean $1 / \mu_{1}$ on the service time $Y_{1}$ at CPU node and continuous distribution with mean $1 / \mu_{2}$ on the service time $Y_{2}$ at I/O node. The levels of $p_{0}$ considered in the simulation study are 0.1 to 0.9 where as levels of $p_{1}$ are 0.9 to 0.1 , where $p_{0}$ is the probability that the job departs from the system and $p_{1}$ is the probability that after service completion at CPU node, the job proceeds to the I/O node. This means with probability $p_{0}=0.1$ the job departs from the system and with probability $p_{1}=0.9$, after service completion at CPU node, the job proceeds to the $\mathrm{I} / \mathrm{O}$ node and so on. Also we have considered the values of $\rho_{1}$ and $\rho_{2}$ such that $\rho_{1}<1$ and $\rho_{2}<1$ for simulation study. Note that in Table 1 wherever $\rho_{1} \geq 1$ and $\rho_{2} \geq 1$ such values of $\rho_{1}$ and $\rho_{2}$ are not considered for simulation study. The intensity parameters $\rho_{1}$ and $\rho_{2}$ are calculated using Equation (1). The different values of $\lambda, \mu_{1}, \mu_{2}$, $p_{0}$ and $p_{1}$ are considered for simulation study as shown in Table 1.

For different levels of $\rho_{1}$, random samples of interarrival times $X_{11}, X_{12}, \cdots, X_{1 n}$ and service times $p_{0} Y_{11}$, 
Table 1. Different levels of intensity parameters considered in the simulation study.

\begin{tabular}{|c|c|c|c|c|c|c|c|c|c|c|c|}
\hline \multicolumn{4}{|c|}{$\lambda=0.1, \mu_{1}=1, \mu_{2}=1$} & \multicolumn{4}{|c|}{$\lambda=0.1, \mu_{1}=1, \mu_{2}=2$} & \multicolumn{4}{|c|}{$\lambda=0.1, \mu_{1}=2, \mu_{2}=1$} \\
\hline$p_{0}$ & $p_{1}$ & $\rho_{1}$ & $\rho_{2}$ & $p_{0}$ & $p_{1}$ & $\rho_{1}$ & $\rho_{2}$ & $p_{0}$ & $p_{1}$ & $\rho_{1}$ & $\rho_{2}$ \\
\hline 0.1 & 0.9 & 1 & 0.9 & 0.1 & 0.9 & 1 & 0.45 & 0.1 & 0.9 & 0.5 & 0.9 \\
\hline 0.2 & 0.8 & 0.5 & 0.4 & 0.2 & 0.8 & 0.5 & 0.2 & 0.2 & 0.8 & 0.25 & 0.4 \\
\hline 0.3 & 0.7 & 0.33 & 0.23 & 0.3 & 0.7 & 0.33 & 0.12 & 0.3 & 0.7 & 0.17 & 0.23 \\
\hline 0.4 & 0.6 & 0.25 & 0.15 & 0.4 & 0.6 & 0.25 & 0.08 & 0.4 & 0.6 & 0.13 & 0.15 \\
\hline 0.5 & 0.5 & 0.2 & 0.1 & 0.5 & 0.5 & 0.2 & 0.05 & 0.5 & 0.5 & 0.1 & 0.1 \\
\hline 0.6 & 0.4 & 0.17 & 0.07 & 0.6 & 0.4 & 0.17 & 0.03 & 0.6 & 0.4 & 0.08 & 0.07 \\
\hline 0.7 & 0.3 & 0.14 & 0.04 & 0.7 & 0.3 & 0.14 & 0.02 & 0.7 & 0.3 & 0.07 & 0.04 \\
\hline 0.8 & 0.2 & 0.13 & 0.03 & 0.8 & 0.2 & 0.13 & 0.01 & 0.8 & 0.2 & 0.06 & 0.03 \\
\hline 0.9 & 0.1 & 0.11 & 0.01 & 0.9 & 0.1 & 0.11 & 0.01 & 0.9 & 0.1 & 0.06 & 0.01 \\
\hline \multicolumn{4}{|c|}{$\lambda=0.5, \mu_{1}=1, \mu_{2}=1$} & \multicolumn{4}{|c|}{$\lambda=0.5, \mu_{1}=1, \mu_{2}=2$} & \multicolumn{4}{|c|}{$\lambda=0.5, \mu_{1}=2, \mu_{2}=1$} \\
\hline$p_{0}$ & $p_{1}$ & $\rho_{1}$ & $\rho_{2}$ & $p_{0}$ & $p_{1}$ & $\rho_{1}$ & $\rho_{2}$ & $p_{0}$ & $p_{1}$ & $\rho_{1}$ & $\rho_{2}$ \\
\hline 0.1 & 0.9 & 5 & 4.5 & 0.1 & 0.9 & 5 & 2.25 & 0.1 & 0.9 & 2.5 & 4.5 \\
\hline 0.2 & 0.8 & 2.5 & 2 & 0.2 & 0.8 & 2.5 & 1 & 0.2 & 0.8 & 1.25 & 2 \\
\hline 0.3 & 0.7 & 1.67 & 1.17 & 0.3 & 0.7 & 1.67 & 0.58 & 0.3 & 0.7 & 0.83 & 1.17 \\
\hline 0.4 & 0.6 & 1.25 & 0.75 & 0.4 & 0.6 & 1.25 & 0.38 & 0.4 & 0.6 & 0.63 & 0.75 \\
\hline 0.5 & 0.5 & 1 & 0.5 & 0.5 & 0.5 & 1 & 0.25 & 0.5 & 0.5 & 0.5 & 0.5 \\
\hline 0.6 & 0.4 & 0.83 & 0.33 & 0.6 & 0.4 & 0.83 & 0.17 & 0.6 & 0.4 & 0.42 & 0.33 \\
\hline 0.7 & 0.3 & 0.71 & 0.21 & 0.7 & 0.3 & 0.71 & 0.11 & 0.7 & 0.3 & 0.36 & 0.21 \\
\hline 0.8 & 0.2 & 0.63 & 0.13 & 0.8 & 0.2 & 0.63 & 0.06 & 0.8 & 0.2 & 0.31 & 0.13 \\
\hline 0.9 & 0.1 & 0.56 & 0.06 & 0.9 & 0.1 & 0.56 & 0.03 & 0.9 & 0.1 & 0.28 & 0.06 \\
\hline \multicolumn{4}{|c|}{$\lambda=0.9, \mu_{1}=1, \mu_{2}=1$} & \multicolumn{4}{|c|}{$\lambda=0.9, \mu_{1}=1, \mu_{2}=2$} & \multicolumn{4}{|c|}{$\lambda=0.9, \mu_{1}=2, \mu_{2}=1$} \\
\hline$p_{0}$ & $p_{1}$ & $\rho_{1}$ & $\rho_{2}$ & $p_{0}$ & $p_{1}$ & $\rho_{1}$ & $\rho_{2}$ & $p_{0}$ & $p_{1}$ & $\rho_{1}$ & $\rho_{2}$ \\
\hline 0.1 & 0.9 & 9 & 8.1 & 0.1 & 0.9 & 9 & 4.05 & 0.1 & 0.9 & 4.5 & 8.1 \\
\hline 0.2 & 0.8 & 4.5 & 3.6 & 0.2 & 0.8 & 4.5 & 1.8 & 0.2 & 0.8 & 2.25 & 3.6 \\
\hline 0.3 & 0.7 & 3 & 2.1 & 0.3 & 0.7 & 3 & 1.05 & 0.3 & 0.7 & 1.5 & 2.1 \\
\hline 0.4 & 0.6 & 2.25 & 1.35 & 0.4 & 0.6 & 2.25 & 0.68 & 0.4 & 0.6 & 1.13 & 1.35 \\
\hline 0.5 & 0.5 & 1.8 & 0.9 & 0.5 & 0.5 & 1.8 & 0.45 & 0.5 & 0.5 & 0.9 & 0.9 \\
\hline 0.6 & 0.4 & 1.5 & 0.6 & 0.6 & 0.4 & 1.5 & 0.3 & 0.6 & 0.4 & 0.75 & 0.6 \\
\hline 0.7 & 0.3 & 1.29 & 0.39 & 0.7 & 0.3 & 1.29 & 0.19 & 0.7 & 0.3 & 0.64 & 0.39 \\
\hline 0.8 & 0.2 & 1.13 & 0.23 & 0.8 & 0.2 & 1.13 & 0.11 & 0.8 & 0.2 & 0.56 & 0.23 \\
\hline 0.9 & 0.1 & 1 & 0.1 & 0.9 & 0.1 & 1 & 0.05 & 0.9 & 0.1 & 0.5 & 0.1 \\
\hline
\end{tabular}


$p_{0} Y_{12}, \cdots, p_{0} Y_{1 n}$ are drawn from $X_{1}$ and $Y_{1}$ respectively. Also for each level of $\rho_{2}$ random samples of inter-arrival times $p_{1} X_{21}, p_{1} X_{22}, \cdots, p_{1} X_{2 m}$ and service times $p_{0} Y_{21}, p_{0} Y_{22}, \cdots, p_{0} Y_{2 m}$ are drawn from $X_{2}$ and $Y_{2}$ respectively. Next $N=1000$ bootstrap resamples each of size $n$ and $m=10,20,29$ are drawn from the original samples, as well as $N=1000 \mathrm{BB}$ replications are simulated for the original samples. According to Equations (10), (11), (15), (19), (23), (27)-(29), (31) and (32) in respective, we obtain CAN1, CAN2, SB1, SB2, BB1, BB2, PB1, PB2, $\mathrm{BCaB} 1$ and $\mathrm{BCaB} 2$ confidence intervals of intensities $\rho_{1}$ and $\rho_{2}$ with confidence level $90 \%$. The above simulation process is replicated $N=1000$ times and we compute coverage percentages, average lengths and relative coverage of the above mentioned confidence intervals. We utilize a PC Dual Core and apply Matlab ${ }^{\circledR} 7.0 .1$ to accomplish all simulations.

Here $M$ represents exponential distribution, $E_{4}$ a 4stage Erlang distribution, $H_{4}^{P e}$ a 4-stage hyper-expo- nential distribution and $H_{4}^{P o}$ a 4-stage hypo-exponential distribution.

Based on the above mentioned interval estimation approaches, the coverage percentage, average lengths and relative coverage of intensities $\rho_{1}$ and $\rho_{2}$ are shown in Tables 3 to $\mathbf{7}$ for queueing network models (presented in Table 2) with short run data, we find that average lengths

Table 2. Different queueing network models simulated for study.

\begin{tabular}{cc}
\hline Queueing Networks & \multicolumn{2}{c}{ Models Simulated } \\
\hline$M / G / 1$ to $G / M / 1$ & $M / E_{4} / 1$ to $E_{4} / M / 1$ \\
& $M / H_{4}^{P_{e}} / 1$ to $H_{4}^{P_{e}} / M / 1$ \\
\hline & $E_{4} / H_{4}^{P_{e}} / 1$ to $H_{4}^{P_{e}} / E_{4} / 1$ \\
& $E_{4} / H_{4}^{P_{o}} / 1$ to $H_{4}^{P_{o}} / E_{4} / 1$ \\
& $H_{4}^{P_{e}} / H_{4}^{P_{o}} / 1$ to $H_{4}^{P_{o}} / H_{4}^{P_{e}} / 1$ \\
\hline
\end{tabular}

Table 3. Simulation results of coverage percentage, average lengths, and relative coverage for $90 \%$ confidence intervals under queueing network. $M / E_{4} / 1$ to $E_{4} / M / 1$.

\begin{tabular}{|c|c|c|c|c|c|c|c|c|c|c|}
\hline \multirow{2}{*}{$\begin{array}{c}\text { Intensity } \\
\text { Parameters }\end{array}$} & \multirow{2}{*}{$\begin{array}{l}\text { Estimation } \\
\text { Approach }\end{array}$} & \multicolumn{3}{|c|}{ Coverage Percentage } & \multicolumn{3}{|c|}{ Average Length } & \multicolumn{3}{|c|}{ Relative Coverage } \\
\hline & & $n=10$ & $n=20$ & $n=29$ & $n=10$ & $n=20$ & $n=29$ & $n=10$ & $n=20$ & $n=29$ \\
\hline \multirow{10}{*}{$\begin{array}{c}p_{0}=0.2 \\
p_{1}=0.8 \\
\rho_{1}=0.5 \\
\text { and } \\
\rho_{2}=0.2\end{array}$} & CAN1 & 0.878 & 0.895 & 0.878 & 0.611 & 0.416 & 0.345 & 1.437 & 2.151 & 2.548 \\
\hline & CAN2 & 0.840 & 0.881 & 0.871 & 0.220 & 0.162 & 0.135 & 3.816 & 5.431 & 6.465 \\
\hline & SB1 & 0.916 & 0.910 & 0.900 & 0.748 & 0.455 & 0.365 & 1.225 & 2.002 & 2.465 \\
\hline & SB2 & 0.835 & 0.875 & 0.869 & 0.217 & 0.161 & 0.134 & 3.843 & 5.425 & 6.480 \\
\hline & BB1 & 0.879 & 0.898 & 0.877 & 0.628 & 0.418 & 0.344 & 1.399 & 2.148 & 2.547 \\
\hline & BB2 & 0.817 & 0.864 & 0.856 & 0.205 & 0.157 & 0.131 & 3.978 & 5.517 & 6.514 \\
\hline & PB1 & 0.832 & 0.874 & 0.867 & 0.687 & 0.438 & 0.356 & 1.211 & 1.994 & 2.437 \\
\hline & PB2 & 0.831 & 0.874 & 0.869 & 0.214 & 0.159 & 0.133 & 3.892 & 5.490 & 6.537 \\
\hline & BCaB1 & 0.831 & 0.877 & 0.872 & 0.668 & 0.433 & 0.353 & 1.243 & 2.026 & 2.473 \\
\hline & BCaB2 & 0.837 & 0.871 & 0.871 & 0.214 & 0.160 & 0.133 & 3.913 & 5.454 & 6.535 \\
\hline \multirow{10}{*}{$\begin{array}{c}p_{0}=0.9 \\
p_{1}=0.1 \\
\rho_{1}=0.11 \\
\text { and } \\
\rho_{2}=0.01\end{array}$} & CAN1 & 0.870 & 0.885 & 0.868 & 0.139 & 0.092 & 0.076 & 6.276 & 9.648 & 11.410 \\
\hline & CAN2 & 0.832 & 0.878 & 0.867 & 0.006 & 0.004 & 0.004 & 134.515 & 195.434 & 232.230 \\
\hline & SB1 & 0.910 & 0.912 & 0.887 & 0.171 & 0.100 & 0.081 & 5.310 & 9.116 & 11.013 \\
\hline & SB2 & 0.827 & 0.879 & 0.866 & 0.006 & 0.004 & 0.004 & 135.068 & 196.738 & 232.756 \\
\hline & BB1 & 0.879 & 0.882 & 0.867 & 0.143 & 0.092 & 0.076 & 6.138 & 9.586 & 11.400 \\
\hline & BB2 & 0.807 & 0.871 & 0.858 & 0.006 & 0.004 & 0.004 & 139.854 & 200.876 & 235.214 \\
\hline & PB1 & 0.824 & 0.871 & 0.859 & 0.156 & 0.096 & 0.078 & 5.270 & 9.031 & 10.946 \\
\hline & PB2 & 0.826 & 0.869 & 0.866 & 0.006 & 0.004 & 0.004 & 137.508 & 197.084 & 235.030 \\
\hline & BCaB1 & 0.833 & 0.872 & 0.868 & 0.152 & 0.095 & 0.078 & 5.478 & 9.169 & 11.159 \\
\hline & BCaB2 & 0.823 & 0.871 & 0.865 & 0.006 & 0.004 & 0.004 & 136.752 & 196.995 & 234.219 \\
\hline
\end{tabular}




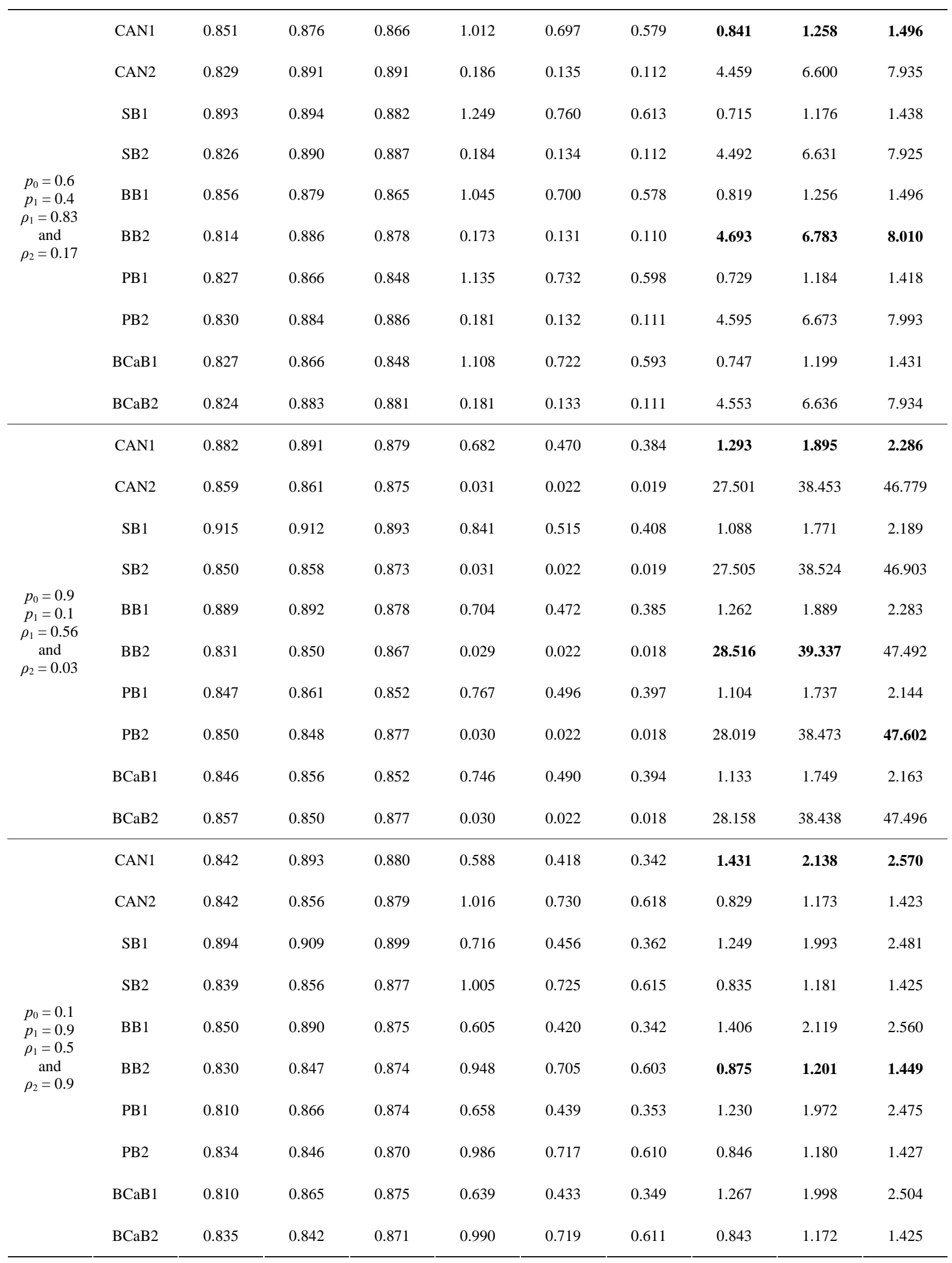


Continued

\begin{tabular}{|c|c|c|c|c|c|c|c|c|c|c|}
\hline & CAN1 & 0.841 & 0.868 & 0.878 & 0.764 & 0.523 & 0.431 & 1.101 & 1.659 & 2.037 \\
\hline \multirow{9}{*}{$\begin{array}{c}p_{0}=0.4 \\
p_{1}=0.6 \\
\rho_{1}=0.63 \\
\text { and } \\
\rho_{2}=0.75\end{array}$} & CAN2 & 0.851 & 0.863 & 0.875 & 0.837 & 0.600 & 0.505 & 1.016 & 1.439 & 1.732 \\
\hline & SB1 & 0.891 & 0.888 & 0.890 & 0.944 & 0.572 & 0.455 & 0.944 & 1.552 & 1.955 \\
\hline & SB2 & 0.850 & 0.859 & 0.875 & 0.828 & 0.596 & 0.503 & 1.026 & 1.440 & 1.739 \\
\hline & BB1 & 0.849 & 0.871 & 0.873 & 0.790 & 0.526 & 0.431 & 1.075 & 1.657 & 2.025 \\
\hline & BB2 & 0.832 & 0.849 & 0.864 & 0.781 & 0.580 & 0.494 & 1.066 & 1.465 & 1.748 \\
\hline & PB1 & 0.810 & 0.849 & 0.863 & 0.864 & 0.551 & 0.444 & 0.937 & 1.540 & 1.943 \\
\hline & PB2 & 0.843 & 0.854 & 0.881 & 0.813 & 0.589 & 0.498 & 1.037 & 1.449 & 1.767 \\
\hline & BCaB1 & 0.817 & 0.853 & 0.862 & 0.840 & 0.544 & 0.441 & 0.973 & 1.569 & 1.957 \\
\hline & BCaB2 & 0.841 & 0.854 & 0.869 & 0.814 & 0.592 & 0.499 & 1.033 & 1.443 & 1.740 \\
\hline \multirow{10}{*}{$\begin{array}{c}p_{0}=0.9 \\
p_{1}=0.1 \\
\rho_{1}=0.28 \\
\text { and } \\
\rho_{2}=0.06\end{array}$} & CAN1 & 0.853 & 0.887 & 0.896 & 0.334 & 0.231 & 0.193 & 2.554 & 3.841 & 4.639 \\
\hline & CAN2 & 0.831 & 0.873 & 0.862 & 0.063 & 0.045 & 0.037 & 13.279 & 19.278 & 23.249 \\
\hline & SB1 & 0.893 & 0.906 & 0.911 & 0.411 & 0.251 & 0.205 & 2.171 & 3.611 & 4.452 \\
\hline & SB2 & 0.828 & 0.869 & 0.859 & 0.062 & 0.045 & 0.037 & 13.405 & 19.311 & 23.232 \\
\hline & BB1 & 0.856 & 0.890 & 0.897 & 0.345 & 0.232 & 0.193 & 2.484 & 3.844 & 4.645 \\
\hline & BB2 & 0.815 & 0.861 & 0.861 & 0.058 & 0.044 & 0.036 & 13.972 & 19.708 & 23.773 \\
\hline & PB1 & 0.824 & 0.858 & 0.877 & 0.377 & 0.242 & 0.199 & 2.187 & 3.546 & 4.398 \\
\hline & PB2 & 0.838 & 0.872 & 0.861 & 0.061 & 0.044 & 0.037 & 13.825 & 19.620 & 23.521 \\
\hline & BCaB1 & 0.830 & 0.857 & 0.880 & 0.364 & 0.238 & 0.198 & 2.278 & 3.595 & 4.449 \\
\hline & $\mathrm{BCaB} 2$ & 0.837 & 0.873 & 0.862 & 0.061 & 0.045 & 0.037 & 13.803 & 19.574 & 23.512 \\
\hline
\end{tabular}

Note that: 1) boldface denotes the greatest relative coverage among the five estimation approach; 2) Confidence intervals of $\rho_{1}$ under different estimation approaches are denoted by CAN1, SB1, BB1, PB1 and BCaB1; 3) Confidence intervals of $\rho_{2}$ under different estimation approaches are denoted by CAN2, SB2, BB2, PB2 and BCaB2.

Table 4. Simulation results of coverage percentage, average lengths, and relative coverage for $90 \%$ confidence intervals under queueing network. $M / H_{4}^{P e} / 1$ to $H_{4}^{P e} / M / 1$.

\begin{tabular}{|c|c|c|c|c|c|c|c|c|c|c|}
\hline \multirow{2}{*}{$\begin{array}{c}\text { Intensity } \\
\text { Parameters }\end{array}$} & \multirow{2}{*}{$\begin{array}{l}\text { Estimation } \\
\text { Approach }\end{array}$} & \multicolumn{3}{|c|}{ Coverage Percentage } & \multicolumn{3}{|c|}{ Average Length } & \multicolumn{3}{|c|}{ Relative Coverage } \\
\hline & & $n=10$ & $n=20$ & $n=29$ & $n=10$ & $n=20$ & $n=29$ & $n=10$ & $n=20$ & $n=29$ \\
\hline \multirow{10}{*}{$\begin{array}{c}p_{0}=0.2 \\
p_{1}=0.8 \\
\rho_{1}=0.5 \\
\text { and } \\
\rho_{2}=0.2\end{array}$} & CAN1 & 0.875 & 0.876 & 0.903 & 0.620 & 0.432 & 0.359 & 1.412 & 2.026 & 2.514 \\
\hline & CAN2 & 0.825 & 0.870 & 0.881 & 0.234 & 0.169 & 0.140 & 3.530 & 5.155 & 6.275 \\
\hline & SB1 & 0.910 & 0.896 & 0.916 & 0.752 & 0.472 & 0.380 & 1.210 & 1.900 & 2.413 \\
\hline & SB2 & 0.826 & 0.869 & 0.882 & 0.235 & 0.169 & 0.140 & 3.512 & 5.147 & 6.279 \\
\hline & BB1 & 0.876 & 0.875 & 0.899 & 0.635 & 0.435 & 0.359 & 1.379 & 2.011 & 2.502 \\
\hline & BB2 & 0.802 & 0.859 & 0.874 & 0.220 & 0.163 & 0.137 & 3.649 & 5.256 & 6.359 \\
\hline & PB1 & 0.845 & 0.874 & 0.895 & 0.688 & 0.454 & 0.371 & 1.228 & 1.923 & 2.414 \\
\hline & PB2 & 0.836 & 0.864 & 0.880 & 0.229 & 0.167 & 0.139 & 3.645 & 5.185 & 6.331 \\
\hline & BCaB1 & 0.841 & 0.875 & 0.891 & 0.669 & 0.448 & 0.367 & 1.257 & 1.951 & 2.427 \\
\hline & BCaB2 & 0.830 & 0.860 & 0.875 & 0.229 & 0.167 & 0.139 & 3.618 & 5.150 & 6.295 \\
\hline
\end{tabular}




\begin{tabular}{|c|c|c|c|c|c|c|c|c|c|c|}
\hline \multirow{10}{*}{$\begin{array}{c}p_{0}=0.9 \\
p_{1}=0.1 \\
\rho_{1}=0.11 \\
\text { and } \\
\rho_{2}=0.01\end{array}$} & CAN1 & 0.887 & 0.900 & 0.885 & 0.140 & 0.097 & 0.080 & 6.315 & 9.299 & 11.041 \\
\hline & CAN2 & 0.824 & 0.866 & 0.884 & 0.006 & 0.005 & 0.004 & 127.283 & 186.510 & 225.524 \\
\hline & SB1 & 0.911 & 0.917 & 0.904 & 0.171 & 0.105 & 0.085 & 5.313 & 8.711 & 10.681 \\
\hline & SB2 & 0.824 & 0.863 & 0.885 & 0.006 & 0.005 & 0.004 & 127.040 & 185.644 & 225.329 \\
\hline & BB1 & 0.894 & 0.901 & 0.885 & 0.145 & 0.097 & 0.080 & 6.166 & 9.256 & 11.057 \\
\hline & BB2 & 0.805 & 0.855 & 0.879 & 0.006 & 0.005 & 0.004 & 132.218 & 189.933 & 229.041 \\
\hline & PB1 & 0.852 & 0.872 & 0.881 & 0.157 & 0.102 & 0.083 & 5.429 & 8.588 & 10.676 \\
\hline & PB2 & 0.815 & 0.851 & 0.890 & 0.006 & 0.005 & 0.004 & 128.530 & 185.849 & 229.244 \\
\hline & BCaB1 & 0.856 & 0.877 & 0.882 & 0.152 & 0.100 & 0.082 & 5.622 & 8.761 & 10.783 \\
\hline & BCaB2 & 0.812 & 0.860 & 0.889 & 0.006 & 0.005 & 0.004 & 127.997 & 187.752 & 228.755 \\
\hline \multirow{10}{*}{$\begin{array}{c}p_{0}=0.6 \\
p_{1}=0.4 \\
\rho_{1}=0.83 \\
\quad \text { and } \\
\rho_{2}=0.17\end{array}$} & CAN1 & 0.871 & 0.883 & 0.876 & 1.082 & 0.730 & 0.599 & 0.805 & 1.209 & 1.463 \\
\hline & CAN2 & 0.834 & 0.873 & 0.897 & 0.199 & 0.143 & 0.117 & 4.186 & 6.097 & 7.688 \\
\hline & SB1 & 0.904 & 0.904 & 0.896 & 1.339 & 0.795 & 0.633 & 0.675 & 1.137 & 1.415 \\
\hline & SB2 & 0.831 & 0.872 & 0.896 & 0.200 & 0.144 & 0.117 & 4.156 & 6.075 & 7.668 \\
\hline & BB1 & 0.879 & 0.884 & 0.878 & 1.118 & 0.734 & 0.599 & 0.786 & 1.205 & 1.467 \\
\hline & BB2 & 0.816 & 0.863 & 0.889 & 0.187 & 0.139 & 0.114 & 4.362 & 6.218 & 7.780 \\
\hline & PB1 & 0.837 & 0.858 & 0.870 & 1.223 & 0.766 & 0.618 & 0.685 & 1.120 & 1.409 \\
\hline & PB2 & 0.824 & 0.869 & 0.895 & 0.195 & 0.142 & 0.116 & 4.219 & 6.140 & 7.746 \\
\hline & BCaB1 & 0.843 & 0.855 & 0.872 & 1.183 & 0.756 & 0.611 & 0.712 & 1.131 & 1.426 \\
\hline & BCaB2 & 0.816 & 0.866 & 0.890 & 0.196 & 0.142 & 0.116 & 4.172 & 6.110 & 7.690 \\
\hline \multirow{10}{*}{$\begin{array}{c}p_{0}=0.9 \\
p_{1}=0.1 \\
\rho_{1}=0.56 \\
\quad \text { and } \\
\rho_{2}=0.03\end{array}$} & CAN1 & 0.866 & 0.880 & 0.874 & 0.700 & 0.489 & 0.399 & 1.238 & 1.800 & 2.192 \\
\hline & CAN2 & 0.837 & 0.868 & 0.894 & 0.032 & 0.023 & 0.020 & 26.164 & 37.301 & 44.908 \\
\hline & SB1 & 0.897 & 0.900 & 0.896 & 0.855 & 0.532 & 0.421 & 1.049 & 1.691 & 2.126 \\
\hline & SB2 & 0.828 & 0.871 & 0.898 & 0.032 & 0.023 & 0.020 & 25.819 & 37.366 & 44.974 \\
\hline & BB1 & 0.870 & 0.881 & 0.880 & 0.720 & 0.492 & 0.398 & 1.209 & 1.790 & 2.211 \\
\hline & BB2 & 0.807 & 0.860 & 0.889 & 0.030 & 0.023 & 0.019 & 26.877 & 38.147 & 45.675 \\
\hline & PB1 & 0.844 & 0.868 & 0.864 & 0.784 & 0.513 & 0.411 & 1.076 & 1.693 & 2.103 \\
\hline & PB2 & 0.827 & 0.872 & 0.897 & 0.031 & 0.023 & 0.020 & 26.381 & 37.956 & 45.425 \\
\hline & BCaB1 & 0.845 & 0.868 & 0.865 & 0.763 & 0.506 & 0.407 & 1.107 & 1.716 & 2.123 \\
\hline & BCaB2 & 0.824 & 0.870 & 0.892 & 0.031 & 0.023 & 0.020 & 26.212 & 37.782 & 45.098 \\
\hline
\end{tabular}




\section{Continued}

\begin{tabular}{|c|c|c|c|c|c|c|c|c|c|c|}
\hline \multirow{10}{*}{$\begin{array}{c}p_{0}=0.1 \\
p_{1}=0.9 \\
\rho_{1}=0.5 \\
\text { and } \\
\rho_{2}=0.9\end{array}$} & CAN1 & 0.859 & 0.889 & 0.901 & 0.629 & 0.434 & 0.363 & 1.366 & 2.046 & 2.483 \\
\hline & CAN2 & 0.863 & 0.888 & 0.875 & 1.045 & 0.748 & 0.628 & 0.826 & 1.188 & 1.394 \\
\hline & SB1 & 0.908 & 0.911 & 0.918 & 0.776 & 0.473 & 0.384 & 1.170 & 1.927 & 2.391 \\
\hline & SB2 & 0.860 & 0.887 & 0.880 & 1.049 & 0.749 & 0.628 & 0.820 & 1.184 & 1.400 \\
\hline & BB1 & 0.861 & 0.890 & 0.903 & 0.649 & 0.437 & 0.363 & 1.326 & 2.037 & 2.491 \\
\hline & BB2 & 0.845 & 0.875 & 0.865 & 0.981 & 0.724 & 0.614 & 0.861 & 1.208 & 1.409 \\
\hline & PB1 & 0.831 & 0.866 & 0.895 & 0.706 & 0.456 & 0.375 & 1.178 & 1.899 & 2.390 \\
\hline & PB2 & 0.855 & 0.885 & 0.866 & 1.027 & 0.738 & 0.622 & 0.833 & 1.199 & 1.393 \\
\hline & BCaB1 & 0.839 & 0.865 & 0.892 & 0.687 & 0.451 & 0.371 & 1.221 & 1.919 & 2.404 \\
\hline & BCaB2 & 0.851 & 0.886 & 0.868 & 1.026 & 0.739 & 0.622 & 0.829 & 1.198 & 1.395 \\
\hline \multirow{10}{*}{$\begin{array}{c}p_{0}=0.4 \\
p_{1}=0.6 \\
\rho_{1}=0.63 \\
\text { and } \\
\rho_{2}=0.75\end{array}$} & CAN1 & 0.881 & 0.899 & 0.900 & 0.778 & 0.551 & 0.451 & 1.133 & 1.632 & 1.995 \\
\hline & CAN2 & 0.848 & 0.879 & 0.862 & 0.884 & 0.635 & 0.529 & 0.959 & 1.385 & 1.629 \\
\hline & SB1 & 0.909 & 0.920 & 0.913 & 0.954 & 0.601 & 0.478 & 0.953 & 1.532 & 1.912 \\
\hline & SB2 & 0.843 & 0.883 & 0.862 & 0.887 & 0.635 & 0.530 & 0.950 & 1.390 & 1.627 \\
\hline & BB1 & 0.881 & 0.900 & 0.903 & 0.804 & 0.554 & 0.451 & 1.096 & 1.624 & 2.001 \\
\hline & BB2 & 0.831 & 0.871 & 0.856 & 0.831 & 0.616 & 0.518 & 1.000 & 1.415 & 1.652 \\
\hline & PB1 & 0.849 & 0.877 & 0.891 & 0.873 & 0.579 & 0.466 & 0.972 & 1.515 & 1.913 \\
\hline & PB2 & 0.833 & 0.884 & 0.867 & 0.868 & 0.626 & 0.523 & 0.960 & 1.412 & 1.657 \\
\hline & BCaB1 & 0.848 & 0.888 & 0.886 & 0.850 & 0.570 & 0.461 & 0.997 & 1.558 & 1.920 \\
\hline & BCaB2 & 0.831 & 0.874 & 0.883 & 0.868 & 0.628 & 0.524 & 0.957 & 1.392 & 1.685 \\
\hline \multirow{10}{*}{$\begin{array}{c}p_{0}=0.9 \\
p_{1}=0.1 \\
\rho_{1}=0.28 \\
\text { and } \\
\rho_{2}=0.06\end{array}$} & CAN1 & 0.859 & 0.876 & 0.891 & 0.347 & 0.242 & 0.200 & 2.477 & 3.615 & 4.452 \\
\hline & CAN2 & 0.849 & 0.848 & 0.872 & 0.067 & 0.047 & 0.039 & 12.746 & 18.094 & 22.514 \\
\hline & SB1 & 0.894 & 0.898 & 0.902 & 0.422 & 0.264 & 0.212 & 2.117 & 3.402 & 4.263 \\
\hline & SB2 & 0.851 & 0.845 & 0.871 & 0.067 & 0.047 & 0.039 & 12.716 & 18.010 & 22.461 \\
\hline & BB1 & 0.861 & 0.881 & 0.887 & 0.357 & 0.244 & 0.200 & 2.415 & 3.613 & 4.437 \\
\hline & BB2 & 0.831 & 0.845 & 0.867 & 0.063 & 0.045 & 0.038 & 13.279 & 18.605 & 22.872 \\
\hline & PB1 & 0.822 & 0.860 & 0.874 & 0.386 & 0.254 & 0.206 & 2.130 & 3.382 & 4.239 \\
\hline & PB2 & 0.848 & 0.849 & 0.880 & 0.065 & 0.046 & 0.038 & 12.964 & 18.347 & 22.926 \\
\hline & BCaB1 & 0.828 & 0.866 & 0.875 & 0.375 & 0.251 & 0.204 & 2.207 & 3.448 & 4.282 \\
\hline & BCaB2 & 0.849 & 0.849 & 0.882 & 0.066 & 0.046 & 0.038 & 12.959 & 18.325 & 22.919 \\
\hline
\end{tabular}

Note that: 1) boldface denotes the greatest relative coverage among the five estimation approach; 2) Confidence intervals of $\rho_{1}$ under different estimation approaches are denoted by CAN1, SB1, BB1, PB1 and BCaB1; 3) Confidence intervals of $\rho_{2}$ under different estimation approaches are denoted by CAN2, SB2, BB2, PB2 and BCaB2. 
Table 5. Simulation results of coverage percentage, average lengths, and relative coverage for $\mathbf{9 0 \%}$ confidence intervals under queueing network: $E_{4} / H_{4}^{P e} / 1$ to $H_{4}^{P e} / E_{4} / 1$.

\begin{tabular}{|c|c|c|c|c|c|c|c|c|c|c|}
\hline \multirow{2}{*}{$\begin{array}{c}\text { Intensity } \\
\text { Parameters }\end{array}$} & \multirow{2}{*}{$\begin{array}{l}\text { Estimation } \\
\text { Approach }\end{array}$} & \multicolumn{3}{|c|}{ Coverage Percentage } & \multicolumn{3}{|c|}{ Average Length } & \multicolumn{3}{|c|}{ Relative Coverage } \\
\hline & & $n=10$ & $n=20$ & $n=29$ & $n=10$ & $n=20$ & $n=29$ & $n=10$ & $n=20$ & $n=29$ \\
\hline \multirow{10}{*}{$\begin{array}{c}p_{0}=0.2 \\
p_{1}=0.8 \\
\rho_{1}=0.5 \\
\text { and } \\
\rho_{2}=0.2\end{array}$} & CAN1 & 0.881 & 0.873 & 0.885 & 0.402 & 0.286 & 0.236 & 2.193 & 3.051 & 3.754 \\
\hline & CAN2 & 0.875 & 0.888 & 0.897 & 0.161 & 0.113 & 0.094 & 5.441 & 7.837 & 9.518 \\
\hline & SB1 & 0.873 & 0.868 & 0.886 & 0.400 & 0.285 & 0.235 & 2.183 & 3.042 & 3.777 \\
\hline & SB2 & 0.875 & 0.892 & 0.896 & 0.163 & 0.114 & 0.095 & 5.367 & 7.816 & 9.463 \\
\hline & BB1 & 0.851 & 0.861 & 0.879 & 0.375 & 0.277 & 0.230 & 2.268 & 3.111 & 3.817 \\
\hline & BB2 & 0.857 & 0.875 & 0.891 & 0.151 & 0.110 & 0.092 & 5.678 & 7.987 & 9.696 \\
\hline & PB1 & 0.849 & 0.870 & 0.889 & 0.392 & 0.282 & 0.232 & 2.164 & 3.082 & 3.824 \\
\hline & PB2 & 0.853 & 0.879 & 0.881 & 0.159 & 0.113 & 0.094 & 5.352 & 7.796 & 9.393 \\
\hline & BCaB1 & 0.849 & 0.868 & 0.882 & 0.392 & 0.282 & 0.232 & 2.167 & 3.077 & 3.794 \\
\hline & BCaB2 & 0.860 & 0.876 & 0.872 & 0.159 & 0.112 & 0.094 & 5.422 & 7.798 & 9.308 \\
\hline \multirow{10}{*}{$\begin{array}{c}p_{0}=0.9 \\
p_{1}=0.1 \\
\rho_{1}=0.11 \\
\text { and } \\
\rho_{2}=0.01\end{array}$} & CAN1 & 0.871 & 0.878 & 0.893 & 0.090 & 0.063 & 0.053 & 9.722 & 13.893 & 16.935 \\
\hline & CAN2 & 0.856 & 0.891 & 0.888 & 0.004 & 0.003 & 0.003 & 195.200 & 281.820 & 338.563 \\
\hline & SB1 & 0.869 & 0.876 & 0.894 & 0.089 & 0.063 & 0.053 & 9.753 & 13.908 & 17.007 \\
\hline & SB2 & 0.859 & 0.889 & 0.889 & 0.004 & 0.003 & 0.003 & 193.152 & 279.335 & 337.997 \\
\hline & BB1 & 0.849 & 0.862 & 0.886 & 0.084 & 0.061 & 0.051 & 10.148 & 14.137 & 17.206 \\
\hline & BB2 & 0.834 & 0.875 & 0.881 & 0.004 & 0.003 & 0.003 & 201.763 & 286.371 & 344.687 \\
\hline & PB1 & 0.858 & 0.865 & 0.884 & 0.088 & 0.062 & 0.052 & 9.794 & 13.872 & 16.953 \\
\hline & PB2 & 0.848 & 0.891 & 0.878 & 0.004 & 0.003 & 0.003 & 195.057 & 283.504 & 336.941 \\
\hline & BCaB1 & 0.859 & 0.865 & 0.880 & 0.087 & 0.062 & 0.052 & 9.833 & 13.876 & 16.891 \\
\hline & BCaB2 & 0.842 & 0.882 & 0.877 & 0.004 & 0.003 & 0.003 & 194.536 & 281.382 & 337.204 \\
\hline \multirow{10}{*}{$\begin{array}{c}p_{0}=0.6 \\
p_{1}=0.4 \\
\rho_{1}=0.83 \\
\quad \text { and } \\
\rho_{2}=0.17\end{array}$} & CAN1 & 0.878 & 0.874 & 0.870 & 0.661 & 0.471 & 0.395 & 1.329 & 1.855 & 2.203 \\
\hline & CAN2 & 0.849 & 0.882 & 0.889 & 0.132 & 0.094 & 0.080 & 6.432 & 9.341 & 11.167 \\
\hline & SB1 & 0.872 & 0.869 & 0.867 & 0.658 & 0.470 & 0.393 & 1.325 & 1.851 & 2.204 \\
\hline & SB2 & 0.849 & 0.882 & 0.891 & 0.134 & 0.095 & 0.080 & 6.356 & 9.282 & 11.156 \\
\hline & BB1 & 0.847 & 0.854 & 0.859 & 0.617 & 0.455 & 0.385 & 1.372 & 1.875 & 2.230 \\
\hline & BB2 & 0.822 & 0.864 & 0.879 & 0.124 & 0.091 & 0.078 & 6.635 & 9.473 & 11.335 \\
\hline & PB1 & 0.865 & 0.866 & 0.869 & 0.646 & 0.464 & 0.390 & 1.338 & 1.866 & 2.229 \\
\hline & PB2 & 0.843 & 0.869 & 0.883 & 0.131 & 0.094 & 0.079 & 6.454 & 9.279 & 11.154 \\
\hline & BCaB1 & 0.868 & 0.864 & 0.865 & 0.645 & 0.464 & 0.390 & 1.345 & 1.862 & 2.217 \\
\hline & $\mathrm{BCaB} 2$ & 0.839 & 0.868 & 0.875 & 0.130 & 0.093 & 0.079 & 6.464 & 9.291 & 11.078 \\
\hline
\end{tabular}


Continued

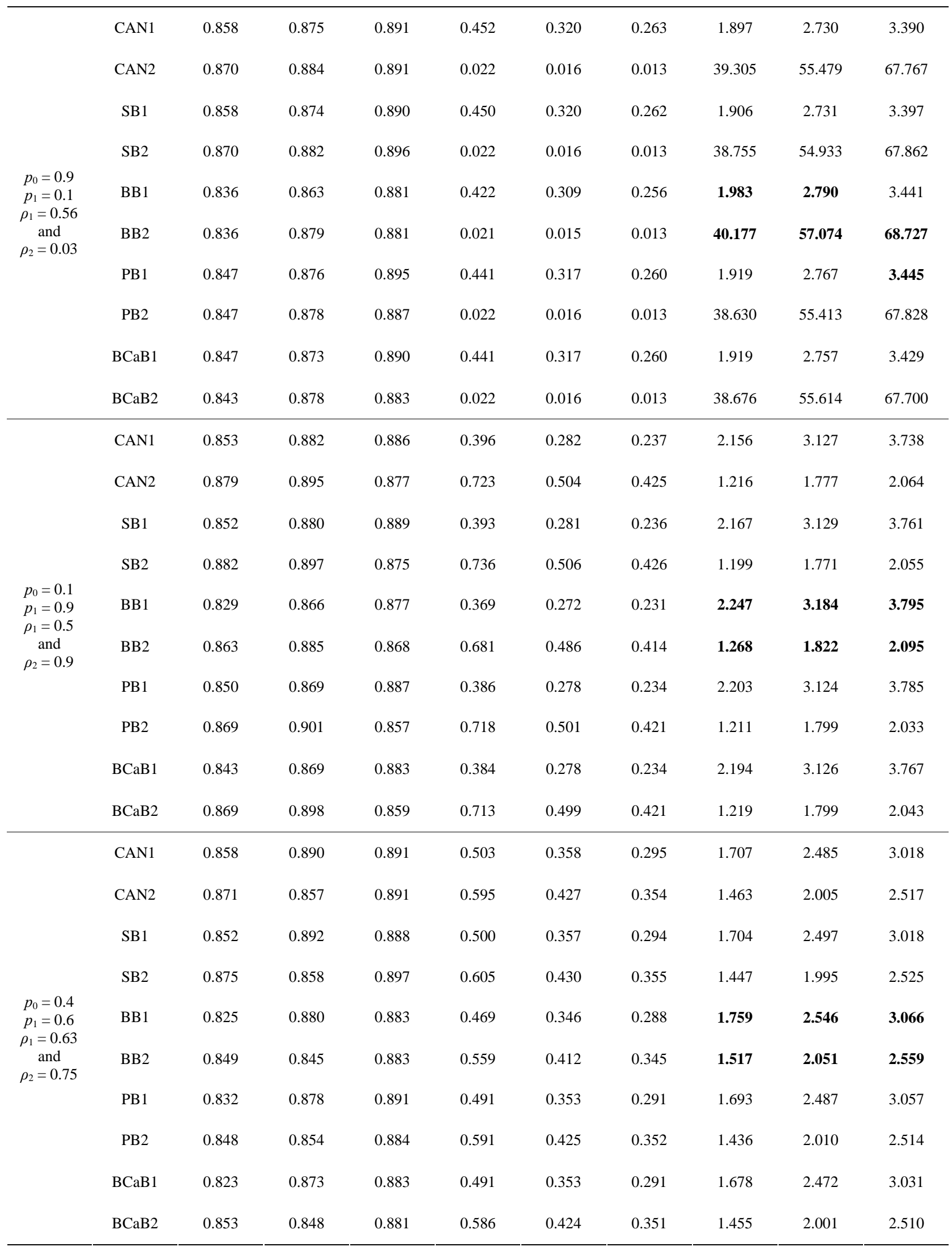




\begin{tabular}{cccccccccccc}
\hline & CAN1 & 0.878 & 0.880 & 0.874 & 0.227 & 0.157 & 0.131 & 3.875 & 5.588 & 6.657 \\
& CAN2 & 0.862 & 0.904 & 0.899 & 0.045 & 0.032 & 0.026 & 19.163 & 28.082 & 34.053 \\
& SB1 & 0.874 & 0.885 & 0.873 & 0.226 & 0.157 & 0.131 & 3.871 & 5.635 & 6.667 \\
& SB2 & 0.864 & 0.903 & 0.897 & 0.046 & 0.032 & 0.027 & 18.942 & 27.925 & 33.823 \\
$\begin{array}{c}p_{0}=0.9 \\
p_{1}=0.1\end{array}$ & BB1 & 0.858 & 0.868 & 0.868 & 0.212 & 0.152 & 0.128 & $\mathbf{4 . 0 5 6}$ & $\mathbf{5 . 7 1 0}$ & $\mathbf{6 . 7 8 1}$ \\
$\begin{array}{c}\rho_{1}=0.28 \\
\text { and }\end{array}$ & BB2 & 0.841 & 0.892 & 0.892 & 0.042 & 0.031 & 0.026 & $\mathbf{1 9 . 8 7 2}$ & $\mathbf{2 8 . 7 0 0}$ & $\mathbf{3 4 . 6 9 1}$ \\
$\rho_{2}=0.06$ & PB1 & 0.864 & 0.869 & 0.870 & 0.222 & 0.155 & 0.130 & 3.898 & 5.595 & 6.704 \\
& PB2 & 0.840 & 0.880 & 0.881 & 0.045 & 0.032 & 0.026 & 18.840 & 27.545 & 33.535 \\
& BCaB1 & 0.862 & 0.874 & 0.866 & 0.221 & 0.155 & 0.130 & 3.909 & 5.637 & 6.681 \\
& BCaB2 & 0.839 & 0.880 & 0.887 & 0.044 & 0.032 & 0.026 & 18.928 & 27.640 & 33.815 \\
\hline
\end{tabular}

Note that: 1) boldface denotes the greatest relative coverage among the five estimation approach; 2) Confidence intervals of $\rho_{1}$ under different estimation approaches are denoted by CAN1, SB1, BB1, PB1 and BCaB1; 3) Confidence intervals of $\rho_{2}$ under different estimation approaches are denoted by CAN2, SB2, BB2, PB2 and BCaB2.

Table 6. Simulation results of coverage percentage, average lengths, and relative coverage for $90 \%$ confidence intervals under queueing network: $E_{4} / H_{4}^{P o} / 1$ to $H_{4}^{P o} / E_{4} / 1$.

\begin{tabular}{|c|c|c|c|c|c|c|c|c|c|c|}
\hline \multirow{2}{*}{$\begin{array}{c}\text { Intensity } \\
\text { Parameters }\end{array}$} & \multirow{2}{*}{$\begin{array}{l}\text { Estimation } \\
\text { Approach }\end{array}$} & \multicolumn{3}{|c|}{ Coverage Percentage } & \multicolumn{3}{|c|}{ Average Length } & \multicolumn{3}{|c|}{ Relative Coverage } \\
\hline & & $n=10$ & $n=20$ & $n=29$ & $n=10$ & $n=20$ & $n=29$ & $n=10$ & $n=20$ & $n=29$ \\
\hline \multirow{10}{*}{$\begin{array}{c}p_{0}=0.2 \\
p_{1}=0.8 \\
\rho_{1}=0.5 \\
\text { and } \\
\rho_{2}=0.2\end{array}$} & CAN1 & 0.868 & 0.889 & 0.892 & 0.400 & 0.283 & 0.236 & 2.173 & 3.140 & 3.775 \\
\hline & CAN2 & 0.863 & 0.883 & 0.877 & 0.159 & 0.115 & 0.094 & 5.421 & 7.646 & 9.316 \\
\hline & SB1 & 0.865 & 0.888 & 0.895 & 0.397 & 0.282 & 0.236 & 2.179 & 3.149 & 3.796 \\
\hline & SB2 & 0.866 & 0.885 & 0.878 & 0.162 & 0.116 & 0.094 & 5.355 & 7.611 & 9.300 \\
\hline & BB1 & 0.845 & 0.883 & 0.883 & 0.373 & 0.273 & 0.230 & 2.268 & 3.240 & 3.835 \\
\hline & BB2 & 0.843 & 0.871 & 0.871 & 0.150 & 0.112 & 0.092 & 5.632 & 7.805 & 9.498 \\
\hline & PB1 & 0.846 & 0.884 & 0.891 & 0.390 & 0.279 & 0.234 & 2.171 & 3.169 & 3.812 \\
\hline & PB2 & 0.850 & 0.875 & 0.866 & 0.158 & 0.115 & 0.094 & 5.379 & 7.626 & 9.256 \\
\hline & $\mathrm{BCaB} 1$ & 0.847 & 0.874 & 0.889 & 0.388 & 0.279 & 0.234 & 2.181 & 3.138 & 3.806 \\
\hline & BCaB2 & 0.848 & 0.872 & 0.867 & 0.157 & 0.114 & 0.093 & 5.388 & 7.632 & 9.285 \\
\hline \multirow{10}{*}{$\begin{array}{c}p_{0}=0.9 \\
p_{1}=0.1 \\
\rho_{1}=0.11 \\
\quad \text { and } \\
\rho_{2}=0.01\end{array}$} & CAN1 & 0.873 & 0.900 & 0.894 & 0.089 & 0.063 & 0.052 & 9.794 & 14.320 & 17.061 \\
\hline & CAN2 & 0.892 & 0.866 & 0.885 & 0.004 & 0.003 & 0.003 & 199.102 & 275.616 & 336.933 \\
\hline & SB1 & 0.874 & 0.899 & 0.891 & 0.089 & 0.063 & 0.052 & 9.846 & 14.339 & 17.045 \\
\hline & SB2 & 0.893 & 0.868 & 0.888 & 0.005 & 0.003 & 0.003 & 196.801 & 274.561 & 336.762 \\
\hline & BB1 & 0.849 & 0.886 & 0.881 & 0.083 & 0.061 & 0.051 & 10.189 & 14.627 & 17.223 \\
\hline & BB2 & 0.873 & 0.859 & 0.874 & 0.004 & 0.003 & 0.003 & 206.607 & 283.242 & 341.123 \\
\hline & PB1 & 0.865 & 0.897 & 0.889 & 0.087 & 0.062 & 0.052 & 9.934 & 14.486 & 17.162 \\
\hline & PB2 & 0.874 & 0.858 & 0.879 & 0.004 & 0.003 & 0.003 & 197.039 & 274.871 & 337.131 \\
\hline & $\mathrm{BCaB} 1$ & 0.863 & 0.897 & 0.885 & 0.087 & 0.062 & 0.052 & 9.929 & 14.497 & 17.097 \\
\hline & BCaB2 & 0.872 & 0.859 & 0.888 & 0.004 & 0.003 & 0.003 & 197.877 & 275.862 & 341.255 \\
\hline
\end{tabular}




\section{Continued}

\begin{tabular}{|c|c|c|c|c|c|c|c|c|c|c|}
\hline \multirow{10}{*}{$\begin{array}{c}p_{0}=0.6 \\
p_{1}=0.4 \\
\rho_{1}=0.83 \\
\text { and } \\
\rho_{2}=0.17\end{array}$} & CAN1 & 0.870 & 0.888 & 0.870 & 0.661 & 0.473 & 0.391 & 1.316 & 1.878 & 2.226 \\
\hline & CAN2 & 0.852 & 0.890 & 0.894 & 0.134 & 0.095 & 0.080 & 6.378 & 9.414 & 11.213 \\
\hline & SB1 & 0.867 & 0.884 & 0.871 & 0.658 & 0.471 & 0.390 & 1.318 & 1.875 & 2.236 \\
\hline & SB2 & 0.859 & 0.891 & 0.893 & 0.135 & 0.095 & 0.080 & 6.347 & 9.388 & 11.170 \\
\hline & BB1 & 0.851 & 0.872 & 0.864 & 0.618 & 0.456 & 0.381 & 1.378 & 1.912 & 2.267 \\
\hline & BB2 & 0.835 & 0.875 & 0.887 & 0.126 & 0.091 & 0.078 & 6.651 & 9.588 & 11.401 \\
\hline & PB1 & 0.863 & 0.886 & 0.868 & 0.646 & 0.466 & 0.386 & 1.336 & 1.901 & 2.247 \\
\hline & PB2 & 0.843 & 0.872 & 0.888 & 0.132 & 0.094 & 0.079 & 6.369 & 9.293 & 11.214 \\
\hline & BCaB1 & 0.862 & 0.885 & 0.863 & 0.645 & 0.466 & 0.386 & 1.336 & 1.900 & 2.236 \\
\hline & BCaB2 & 0.837 & 0.870 & 0.885 & 0.132 & 0.094 & 0.079 & 6.362 & 9.302 & 11.196 \\
\hline \multirow{10}{*}{$\begin{array}{c}p_{0}=0.9 \\
p_{1}=0.1 \\
\rho_{1}=0.56 \\
\text { and } \\
\rho_{2}=0.03\end{array}$} & CAN1 & 0.868 & 0.892 & 0.881 & 0.451 & 0.313 & 0.260 & 1.924 & 2.848 & 3.389 \\
\hline & CAN2 & 0.855 & 0.889 & 0.888 & 0.022 & 0.016 & 0.013 & 38.912 & 56.159 & 67.891 \\
\hline & SB1 & 0.868 & 0.890 & 0.882 & 0.449 & 0.312 & 0.259 & 1.932 & 2.850 & 3.400 \\
\hline & SB2 & 0.855 & 0.886 & 0.889 & 0.022 & 0.016 & 0.013 & 38.389 & 55.660 & 67.803 \\
\hline & BB1 & 0.846 & 0.873 & 0.872 & 0.421 & 0.303 & 0.254 & 2.009 & 2.885 & 3.438 \\
\hline & BB2 & 0.837 & 0.871 & 0.881 & 0.021 & 0.015 & 0.013 & 40.525 & 56.991 & 69.056 \\
\hline & PB1 & 0.852 & 0.885 & 0.871 & 0.441 & 0.309 & 0.257 & 1.931 & 2.868 & 3.388 \\
\hline & PB2 & 0.841 & 0.891 & 0.884 & 0.022 & 0.016 & 0.013 & 38.677 & 56.728 & 68.080 \\
\hline & BCaB1 & 0.853 & 0.878 & 0.872 & 0.440 & 0.308 & 0.257 & 1.940 & 2.846 & 3.390 \\
\hline & BCaB2 & 0.839 & 0.892 & 0.886 & 0.022 & 0.016 & 0.013 & 38.827 & 56.980 & 68.421 \\
\hline \multirow{10}{*}{$\begin{array}{c}p_{0}=0.1 \\
p_{1}=0.9 \\
\rho_{1}=0.5 \\
\text { and } \\
\rho_{2}=0.9\end{array}$} & CAN1 & 0.856 & 0.889 & 0.896 & 0.393 & 0.283 & 0.239 & 2.178 & 3.137 & 3.754 \\
\hline & CAN2 & 0.866 & 0.895 & 0.888 & 0.727 & 0.508 & 0.427 & 1.191 & 1.763 & 2.081 \\
\hline & SB1 & 0.851 & 0.891 & 0.896 & 0.391 & 0.283 & 0.238 & 2.174 & 3.152 & 3.759 \\
\hline & SB2 & 0.862 & 0.889 & 0.889 & 0.738 & 0.510 & 0.428 & 1.168 & 1.742 & 2.077 \\
\hline & BB1 & 0.829 & 0.878 & 0.886 & 0.367 & 0.274 & 0.232 & 2.259 & 3.206 & 3.816 \\
\hline & BB2 & 0.838 & 0.882 & 0.882 & 0.684 & 0.490 & 0.416 & 1.225 & 1.798 & 2.121 \\
\hline & PB1 & 0.851 & 0.888 & 0.882 & 0.385 & 0.279 & 0.236 & 2.213 & 3.179 & 3.731 \\
\hline & PB2 & 0.836 & 0.876 & 0.874 & 0.722 & 0.504 & 0.424 & 1.158 & 1.739 & 2.064 \\
\hline & BCaB1 & 0.848 & 0.881 & 0.885 & 0.384 & 0.279 & 0.236 & 2.210 & 3.155 & 3.746 \\
\hline & BCaB2 & 0.840 & 0.875 & 0.880 & 0.716 & 0.502 & 0.423 & 1.173 & 1.742 & 2.083 \\
\hline
\end{tabular}




\begin{tabular}{|c|c|c|c|c|c|c|c|c|c|c|}
\hline \multirow{10}{*}{$\begin{array}{c}p_{0}=0.4 \\
p_{1}=0.6 \\
\rho_{1}=0.63 \\
\text { and } \\
\rho_{2}=0.75\end{array}$} & CAN1 & 0.864 & 0.901 & 0.904 & 0.510 & 0.362 & 0.294 & 1.694 & 2.486 & 3.076 \\
\hline & CAN2 & 0.878 & 0.889 & 0.896 & 0.607 & 0.424 & 0.351 & 1.447 & 2.096 & 2.551 \\
\hline & SB1 & 0.860 & 0.897 & 0.898 & 0.508 & 0.361 & 0.293 & 1.692 & 2.482 & 3.064 \\
\hline & SB2 & 0.879 & 0.889 & 0.898 & 0.618 & 0.426 & 0.353 & 1.423 & 2.086 & 2.545 \\
\hline & BB1 & 0.844 & 0.884 & 0.894 & 0.477 & 0.350 & 0.286 & 1.769 & 2.526 & 3.121 \\
\hline & BB2 & 0.863 & 0.876 & 0.885 & 0.571 & 0.410 & 0.342 & 1.511 & 2.139 & 2.586 \\
\hline & PB1 & 0.841 & 0.883 & 0.891 & 0.498 & 0.357 & 0.291 & 1.688 & 2.472 & 3.066 \\
\hline & PB2 & 0.857 & 0.882 & 0.885 & 0.602 & 0.421 & 0.350 & 1.424 & 2.096 & 2.530 \\
\hline & BCaB1 & 0.850 & 0.880 & 0.895 & 0.497 & 0.357 & 0.291 & 1.711 & 2.465 & 3.078 \\
\hline & BCaB2 & 0.854 & 0.876 & 0.886 & 0.599 & 0.420 & 0.349 & 1.426 & 2.084 & 2.541 \\
\hline \multirow{10}{*}{$\begin{array}{c}p_{0}=0.9 \\
p_{1}=0.1 \\
\rho_{1}=0.28 \\
\quad \text { and } \\
\rho_{2}=0.06\end{array}$} & CAN1 & 0.867 & 0.880 & 0.884 & 0.216 & 0.159 & 0.130 & 4.009 & 5.550 & 6.780 \\
\hline & CAN2 & 0.897 & 0.894 & 0.893 & 0.045 & 0.031 & 0.027 & 20.111 & 28.392 & 33.686 \\
\hline & SB1 & 0.860 & 0.879 & 0.885 & 0.215 & 0.158 & 0.130 & 4.005 & 5.556 & 6.806 \\
\hline & SB2 & 0.896 & 0.891 & 0.897 & 0.045 & 0.032 & 0.027 & 19.852 & 28.156 & 33.765 \\
\hline & BB1 & 0.846 & 0.865 & 0.878 & 0.202 & 0.153 & 0.127 & 4.193 & 5.647 & 6.907 \\
\hline & BB2 & 0.875 & 0.880 & 0.880 & 0.042 & 0.030 & 0.026 & 20.922 & 28.966 & 34.057 \\
\hline & PB1 & 0.850 & 0.876 & 0.888 & 0.211 & 0.156 & 0.129 & 4.029 & 5.600 & 6.893 \\
\hline & PB2 & 0.874 & 0.879 & 0.896 & 0.044 & 0.031 & 0.026 & 19.771 & 28.134 & 34.043 \\
\hline & BCaB1 & 0.849 & 0.871 & 0.883 & 0.211 & 0.156 & 0.129 & 4.033 & 5.575 & 6.860 \\
\hline & $\mathrm{BCaB} 2$ & 0.874 & 0.873 & 0.893 & 0.044 & 0.031 & 0.026 & 19.880 & 28.019 & 34.021 \\
\hline
\end{tabular}

Note that: 1) boldface denotes the greatest relative coverage among the five estimation approach; 2) Confidence intervals of $\rho_{1}$ under different estimation approaches are denoted by CAN1, SB1, BB1, PB1 and BCaB1; 3) Confidence intervals of $\rho_{2}$ under different estimation approaches are denoted by CAN2, SB2, BB2, PB2 and BCaB2.

Table 7. Simulation results of coverage percentage, average lengths, and relative coverage for $90 \%$ confidence intervals under queueing network: $H_{4}^{P e} / H_{4}^{P o} / 1$ to $H_{4}^{P o} / H_{4}^{P e} / 1$.

\begin{tabular}{|c|c|c|c|c|c|c|c|c|c|c|}
\hline \multirow{2}{*}{$\begin{array}{c}\text { Intensity } \\
\text { Parameters }\end{array}$} & \multirow{2}{*}{$\begin{array}{l}\text { Estimation } \\
\text { Approach }\end{array}$} & \multicolumn{3}{|c|}{ Coverage Percentage } & \multicolumn{3}{|c|}{ Average Length } & \multicolumn{3}{|c|}{ Relative Coverage } \\
\hline & & $n=10$ & $n=20$ & $n=29$ & $n=10$ & $n=20$ & $n=29$ & $n=10$ & $n=20$ & $n=29$ \\
\hline \multirow{9}{*}{$\begin{array}{c}p_{0}=0.2 \\
p_{1}=0.8 \\
\rho_{1}=0.5 \\
\text { and } \\
\rho_{2}=0.2\end{array}$} & CAN1 & 0.858 & 0.858 & 0.901 & 0.430 & 0.306 & 0.256 & 1.993 & 2.799 & 3.518 \\
\hline & CAN2 & 0.864 & 0.870 & 0.895 & 0.174 & 0.122 & 0.102 & 4.977 & 7.119 & 8.803 \\
\hline & SB1 & 0.863 & 0.864 & 0.905 & 0.436 & 0.308 & 0.256 & 1.980 & 2.808 & 3.529 \\
\hline & SB2 & 0.869 & 0.872 & 0.899 & 0.175 & 0.123 & 0.102 & 4.952 & 7.101 & 8.821 \\
\hline & BB1 & 0.839 & 0.845 & 0.897 & 0.405 & 0.296 & 0.250 & 2.071 & 2.855 & 3.592 \\
\hline & BB2 & 0.841 & 0.857 & 0.886 & 0.163 & 0.118 & 0.099 & 5.147 & 7.256 & 8.922 \\
\hline & PB1 & 0.860 & 0.854 & 0.892 & 0.426 & 0.304 & 0.254 & 2.017 & 2.813 & 3.513 \\
\hline & BCaB1 & 0.859 & 0.850 & 0.886 & 0.424 & 0.303 & 0.253 & 2.025 & 2.809 & 3.495 \\
\hline & BCaB2 & 0.846 & 0.868 & 0.881 & 0.170 & 0.121 & 0.101 & 4.966 & 7.180 & 8.740 \\
\hline
\end{tabular}




\section{Continued}

\begin{tabular}{|c|c|c|c|c|c|c|c|c|c|c|}
\hline \multirow{10}{*}{$\begin{array}{c}p_{0}=0.9 \\
p_{1}=0.1 \\
\rho_{1}=0.11 \\
\text { and } \\
\rho_{2}=0.01\end{array}$} & CAN1 & 0.859 & 0.869 & 0.903 & 0.096 & 0.069 & 0.057 & 8.959 & 12.609 & 15.900 \\
\hline & CAN2 & 0.863 & 0.875 & 0.899 & 0.005 & 0.003 & 0.003 & 178.166 & 256.808 & 313.751 \\
\hline & SB1 & 0.861 & 0.872 & 0.902 & 0.097 & 0.069 & 0.057 & 8.882 & 12.572 & 15.840 \\
\hline & SB2 & 0.871 & 0.874 & 0.898 & 0.005 & 0.003 & 0.003 & 177.672 & 255.224 & 312.522 \\
\hline & BB1 & 0.840 & 0.857 & 0.896 & 0.090 & 0.067 & 0.055 & 9.318 & 12.869 & 16.171 \\
\hline & BB2 & 0.844 & 0.865 & 0.895 & 0.005 & 0.003 & 0.003 & 185.562 & 262.597 & 320.580 \\
\hline & PB1 & 0.840 & 0.857 & 0.906 & 0.095 & 0.068 & 0.056 & 8.868 & 12.521 & 16.068 \\
\hline & PB2 & 0.852 & 0.868 & 0.899 & 0.005 & 0.003 & 0.003 & 177.949 & 256.892 & 316.192 \\
\hline & BCaB1 & 0.844 & 0.862 & 0.902 & 0.094 & 0.068 & 0.056 & 8.948 & 12.625 & 16.029 \\
\hline & BCaB2 & 0.850 & 0.861 & 0.899 & 0.005 & 0.003 & 0.003 & 178.207 & 255.533 & 316.061 \\
\hline \multirow{10}{*}{$\begin{array}{c}p_{0}=0.6 \\
p_{1}=0.4 \\
\rho_{1}=0.83 \\
\text { and } \\
\rho_{2}=0.17\end{array}$} & CAN1 & 0.857 & 0.877 & 0.892 & 0.727 & 0.512 & 0.430 & 1.178 & 1.712 & 2.076 \\
\hline & CAN2 & 0.879 & 0.892 & 0.904 & 0.143 & 0.102 & 0.084 & 6.127 & 8.703 & 10.757 \\
\hline & SB1 & 0.864 & 0.878 & 0.894 & 0.737 & 0.513 & 0.431 & 1.173 & 1.710 & 2.073 \\
\hline & SB2 & 0.879 & 0.892 & 0.907 & 0.145 & 0.103 & 0.084 & 6.058 & 8.655 & 10.759 \\
\hline & BB1 & 0.842 & 0.865 & 0.886 & 0.684 & 0.495 & 0.419 & 1.230 & 1.747 & 2.115 \\
\hline & BB2 & 0.852 & 0.883 & 0.897 & 0.135 & 0.099 & 0.082 & 6.312 & 8.894 & 10.924 \\
\hline & PB1 & 0.849 & 0.859 & 0.883 & 0.720 & 0.507 & 0.427 & 1.179 & 1.696 & 2.067 \\
\hline & PB2 & 0.855 & 0.885 & 0.911 & 0.142 & 0.102 & 0.083 & 6.031 & 8.703 & 10.910 \\
\hline & BCaB1 & 0.840 & 0.860 & 0.882 & 0.716 & 0.505 & 0.426 & 1.173 & 1.702 & 2.069 \\
\hline & BCaB2 & 0.848 & 0.879 & 0.911 & 0.141 & 0.101 & 0.083 & 6.012 & 8.661 & 10.941 \\
\hline \multirow{10}{*}{$\begin{array}{c}p_{0}=0.9 \\
p_{1}=0.1 \\
\rho_{1}=0.56 \\
\text { and } \\
\rho_{2}=0.03\end{array}$} & CAN1 & 0.867 & 0.883 & 0.881 & 0.475 & 0.340 & 0.281 & 1.826 & 2.598 & 3.138 \\
\hline & CAN2 & 0.868 & 0.883 & 0.874 & 0.024 & 0.017 & 0.014 & 36.033 & 52.147 & 62.267 \\
\hline & SB1 & 0.865 & 0.886 & 0.885 & 0.482 & 0.342 & 0.281 & 1.795 & 2.593 & 3.147 \\
\hline & SB2 & 0.869 & 0.887 & 0.871 & 0.024 & 0.017 & 0.014 & 35.563 & 52.065 & 61.943 \\
\hline & BB1 & 0.847 & 0.871 & 0.872 & 0.447 & 0.329 & 0.274 & 1.896 & 2.650 & 3.186 \\
\hline & BB2 & 0.843 & 0.871 & 0.864 & 0.023 & 0.016 & 0.014 & 37.101 & 53.150 & 63.192 \\
\hline & PB1 & 0.863 & 0.870 & 0.884 & 0.471 & 0.337 & 0.278 & 1.833 & 2.579 & 3.176 \\
\hline & PB2 & 0.857 & 0.878 & 0.872 & 0.024 & 0.017 & 0.014 & 35.875 & 52.258 & 62.681 \\
\hline & BCaB1 & 0.856 & 0.873 & 0.876 & 0.468 & 0.336 & 0.278 & 1.831 & 2.598 & 3.153 \\
\hline & BCaB2 & 0.860 & 0.874 & 0.866 & 0.024 & 0.017 & 0.014 & 36.209 & 52.085 & 62.304 \\
\hline
\end{tabular}




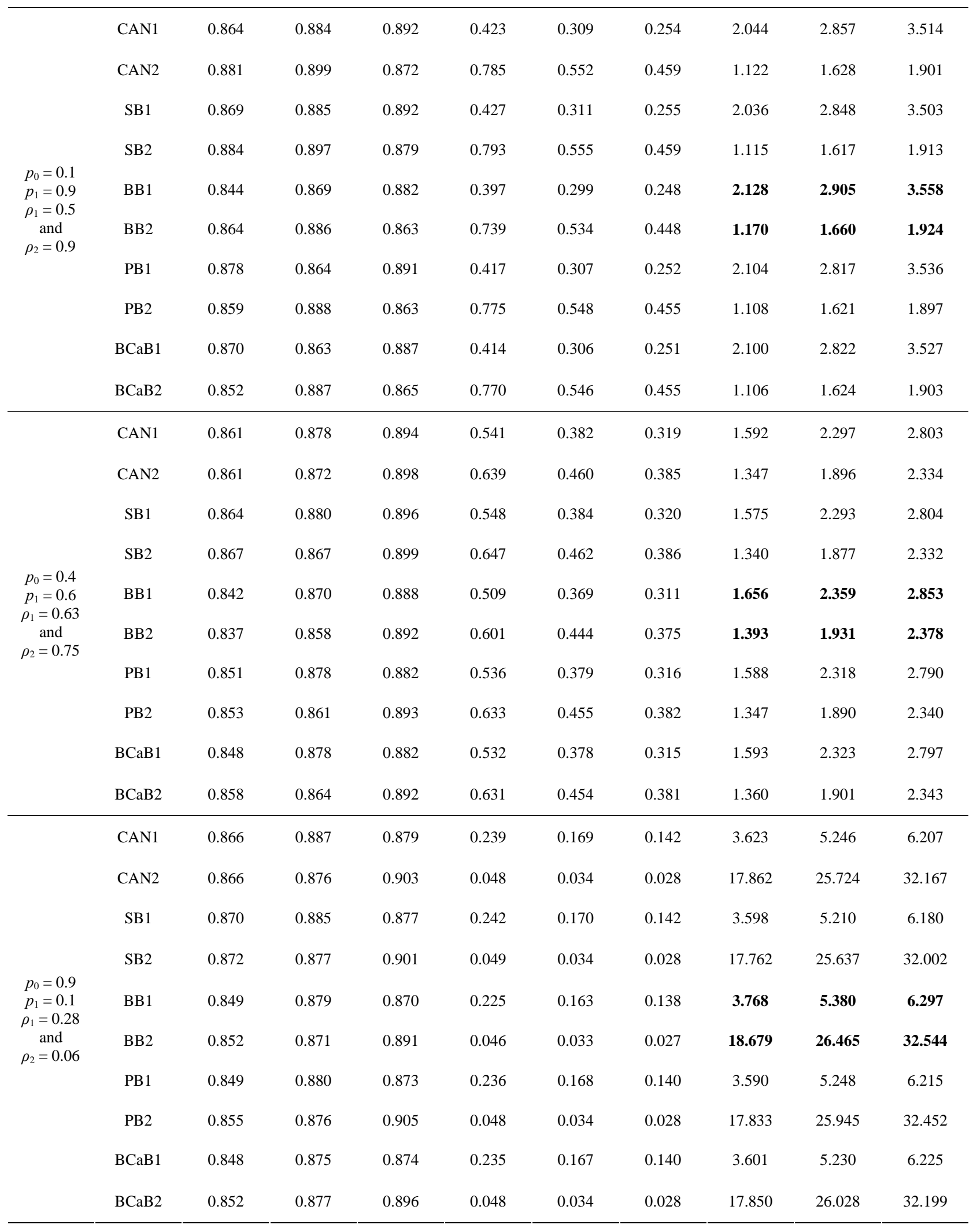

Note that: 1) boldface denotes the greatest relative coverage among the five estimation approach; 2) Confidence intervals of $\rho_{1}$ under different estimation approaches are denoted by CAN1, SB1, BB1, PB1 and BCaB1; 3) Confidence intervals of $\rho_{2}$ under different estimation approaches are denoted by CAN2, SB2, BB2, PB2 and BCaB2. 
are decreasing as $p_{0}$ approaches 1 ( $p_{1}$ approaches 0$)$ but both coverage percentages and relative coverage are increasing as $p_{0}$ approaches 1 ( $p_{1}$ approaches 0$)$.

Also we find that average lengths are decreasing with sample size $n$ but both coverage percentages and relative coverage are increasing with sample size $n$. From Tables 3 to 7 one can observe that the coverage percentage can approach to $90 \%$ when $n$ increases up to 29 .

1) In queueing network models $M / E_{4} / 1$ to $E_{4} / M / 1$ and $M / H_{4}^{P e} / 1$ to $H_{4}^{P e} / M / 1$, the estimation approach CAN and $\mathrm{BB}$ has the greatest relative coverage among the five confidence intervals for $\rho_{1}$ and $\rho_{2}$ respectively for different values of $p_{0}$ and $p_{1}$.

2) In queueing network models $E_{4} / H_{4}^{P e} / 1$ to $H_{4}^{P e} / E_{4} / 1, \quad E_{4} / H_{4}^{P o} / 1$ to $H_{4}^{P o} / E_{4} / 1$ and $H_{4}^{P e} / H_{4}^{P o} / 1$ to $H_{4}^{P o} / H_{4}^{P e} / 1$, the estimation approach $\mathrm{BB}$ has the greatest relative coverage among the five confidence intervals for $\rho_{1}$ and $\rho_{2}$ for different values of $p_{0}$ and $p_{1}$.

3) Average lengths are decreasing as sample size $n$ increases for both $\rho_{1}$ and $\rho_{2}$. Also relative coverage increases with $\mathrm{n}$ for $\rho_{1}$ and $\rho_{2}$.

4) Some poor coverage percentage of above confidence intervals with respect to the nominal level $90 \%$ may be due to small sample size $n$.

\section{Conclusion}

This paper provides the interval estimations of intensities $\rho_{1}$ and $\rho_{2}$ for an open queueing network with feedback. Different estimation approaches CAN, SB, BB, PB and $\mathrm{BCaB}$ are applied to produce confidence intervals for intensities $\rho_{1}$ and $\rho_{2}$. The relative coverage is adopted to understand, compare and assess performance of the resulted confidence intervals. From simulation study it is clear that CAN and BB method has the best performance on interval estimation of intensities $\rho_{1}$ and $\rho_{2}$ for $M / G / 1$ to $M / G / 1$ queueing network and BB method has the best performance on interval estimation of intensities $\rho_{1}$ and $\rho_{2}$ for $G / G / 1$ to $G / G / 1$ queueing network with short run data. And approach is easily applied to practical queueing network system such as all types of open, closed, mixed queueing networks as well as cyclic, retrial queueing models.

\section{REFERENCES}

[1] R. L. Disney, "Random Flow in Queueing Networks: A Review and a Critique," A.I.E.E. Transactions, Vol. 8, No. 1, 1975, pp. 268-288.

[2] P. J. Burke, "Proof of Conjecture on the Inter-Arrival Time Distribution in M/M/1 Queue with Feedback," IEEE Transactions on Communications, Vol. 24, No. 5, 1976, pp. 175-178. doi:10.1109/TCOM.1976.1093335

[3] F.J. Beautler and B. Melamed, "Decomposition and Customer Streams of Feedback Networks of Queues in Equi- librium," Operation Research, Vol. 26, No. 6, 1978, pp. 1059-1072. doi:10.1287/opre.26.6.1059

[4] J. R. Jackson, "Networks of Waiting Lines," Operations Research, Vol. 5, No. 4, 1957, pp. 518-521. doi:10.1287/opre.5.4.518

[5] B. Simon and R. D. Foley, "Some Results on Sojourn Times in Acyclic Jackson Network," Management Science, Vol. 25, No. 10, 1979, pp. 1027-1034. doi:10.1287/mnsc.25.10.1027

[6] B. Melamed, "Sojourn Times in Queueing Networks," Technical Report, Department of Industrial Engineering and Management Sciences, Northwestern University, Evanston, 1980 .

[7] R. L. Disney and P. C. Kiessler, "Traffic Processes in Queueing Networks: A Markov Renewal Approach,” Johns Hopkins University Press, Baltimore, 1987.

[8] D. Thiruvaiyaru, I. V. Basava and U. N. Bhat, "Estimation for a Class of Simple Queueing Network," Queueing Systems, Vol. 9, No. 3, 1991, pp. 301-312. doi:10.1007/BF01158468

[9] D. Thiruvaiyaru and I. V. Basava, "Maximum Likelihood Estimation for Queueing Networks,” In: B. L. S. Prakasa Rao and B. R. Bhat, Eds., Stochastic Processes and Statistical Inference, New Age International Publications, New Delhi, 1996, pp. 132-149.

[10] L. Kleinrock, “Queueing Systems, Vol. II: Computer Applications,” John Wiley \& Sons, New York, 1976.

[11] P. J. Denning and J. P. Buzen, "The Operational Analysis of Queueing Network Models,” ACM Computing Surveys, Vol. 10, No. 3, 1978, pp. 225-261.

[12] B. Efron, "Bootstrap Methods: Another Look at the Jackknife,” Annals of Statistics, Vol. 7, No. 1, 1979, pp. 1-26. doi:10.1214/aos/1176344552

[13] B. Efron, "The Jackknife, the Bootstrap, and Other Resampling Plans,” CBMS-NSF Regional Conference Series in Applied Mathematics, Monograph 38, SIAM, Philadelphia, 1982.

[14] B. Efron, "Better Bootstrap Confidence Intervals,” Journal of the American Statistical Association, Vol. 82, No. 397, 1987, pp. 171-200. doi:10.2307/2289144

[15] D. B. Rubin, “The Bayesian Bootstrap," The Annals of Statistics, Vol. 9, No. 1, 1981, pp. 130-134. doi:10.1214/aos/1176345338

[16] R. G. Miller, “The Jackknife: A Review,” Biometrika, Vol. 61, No. 1, 1974, pp. 1-15.

[17] Y.-K. Chu and J.-C. Ke, "Confidence Intervals of Mean Response Time for an M/G/1 Queueing System: Bootstrap Simulation," Applied Mathematics and Computation, Vol. 180, No. 1, 2006, pp. 255-263. doi:10.1016/j.amc.2005.11.145

[18] Y. K. Chu and J.C. Ke, Interval Estimation of Mean Response Time for a G/M/1 Queueing System: Empirical Laplace Function Approach,” Mathematical Methods in the Applied Sciences, Vol. 30, No. 6, 2006, pp. 707-715. doi:10.1002/mma.806

[19] J. C. Ke and Y. K. Chu, "Nonparametric and Simulated Analysis of Intensity for Queueing System,” Applied 
Mathematics and Computation, Vol. 183, No. 2, 2006, pp. 1280-1291. doi:10.1016/j.amc.2006.05.163

[20] J. C. Ke and Y. K. Chu, "Comparison on Five Estimation Approaches of Intensity for a Queueing System with Short Run,” Computational Statistics, Vol. 24, No. 4, 2009, pp.
567-582.

[21] R. V. Hogg, A. T. Craig and J. W. McKean, "Introduction to Mathematical Statistics,” 6th Edition, Prentice-Hall, Inc., Upper Saddle River, 2011. 